\title{
Measuring the galaxy power spectrum with multiresolution decomposition - III. velocity field analysis
}

\author{
XiaoHu Yang ${ }^{1,2}$ Long-Long Feng ${ }^{1,2,3} \quad$ YaoQuan Chu ${ }^{1,2} \quad$ Li-Zhi Fang ${ }^{4}$
}

Received __; accepted

\footnotetext{
${ }^{1}$ Center for Astrophysics, University of Science and Technology of China, Hefei, Anhui 230026,P.R.China

${ }^{2}$ National Astronomical Observatories, Chinese Academy of Science, Chao-Yang District, Beijing, 100012, P.R. China

${ }^{3}$ Institute for Theoretical Physics, Academic of Science, Beijing, 100080, P.R. China

${ }^{4}$ Department of Physics, University of Arizona, Tucson, AZ 85721
} 


\begin{abstract}
In this paper, we develop the method of analyzing the velocity field of cosmic matter with a multiresolution decomposition. This is necessary in calculating the redshift distortion of power spectrum in the discrete wavelet transform (DWT) representation. We show that, in the DWT analysis, the velocity field can be described by discrete variables, which are given by assignment of the number density and velocity into the DWT modes. These DWT variables are complete and not redundant. In this scheme, the peculiar velocity and pairwise velocity of galaxies or particles are given by field variables. As a consequence, the velocity dispersion (VD) and pairwise velocity dispersion (PVD) are no longer measured by number-counting or pair-counting statistic, but with the ensemble of the field variables, and therefore, they are free from the bias due to the number-counting and pair-counting. We analyzed the VD and PVD of the velocity fields given by the N-body simulation for models of the SCDM, $\tau \mathrm{CDM}$ and $\Lambda \mathrm{CDM}$. The spectrum (scale-dependence) of the VD and PVD show that the length scale of the two-point correlation of the velocity field is as large as few tens $\mathrm{h}^{-1}$ Mpc. Although the VD and PVD show similar behavior in some aspects, they are substantially different from each other. The VD-to-PVD ratio shows the difference between the scale-dependencies of the VD and PVD. More prominent difference between the VD and PVD is shown by probability distribution function. The one-point distribution of peculiar velocity is approximately exponential, while the pairwise velocity's is lognormal, i.e. of long tail. This difference indicates that the cosmic velocity field is typically intermittent.
\end{abstract}

Subject headings: cosmology: theory - large-scale structure of universe 


\section{Introduction}

The radial (redshift) distance of galaxies is distorted by their peculiar velocity. Observation of redshift survey provides only a distorted picture of the galaxy spatial distribution in the radial direction. The galaxy power spectrum $P^{S}(k)$ in the Fourier representation is measured from the redshift-distorted galaxy distribution. It is the power spectrum in redshift space. To recover the power spectrum in real space, $P^{R}(k)$, one needs to map the distributions of galaxies from the redshift space to the real space. Semi-phenomenological theory of redshift distortion show that the mapping between redshift and real spaces gives

$$
P^{S}(k)=G(k) P^{R}(k),
$$

where redshift distortion factor $G(k)$ depends on two parameters: redshift distortion parameter $\beta$, and 1-D peculiar velocity dispersion (VD) $\sigma^{v}$. This leads to two effects: 1 . the enhancing of power on large scales due to the linear effect of redshift distortion; 2. the suppressing of power on small scales due to the random motions of galaxies inside virialized groups and clusters of galaxies.

In deriving the relation (1), the following assumptions are employed: 1 . the effect of the coupling between the peculiar velocity and the density perturbations is linear (Kaiser 1987); 2 . the effect of the random motions of galaxies is independent of the density perturbations of cosmic mass field, 3. the probability distribution function (PDF) of random peculiar velocity or the pairwise velocity is either gaussian or exponential. Therefore, besides the linear effect, or point 1, eq.(1) does not require the information of the spatial distribution of peculiar velocity of galaxies (or dark matter). The parameter $\sigma^{v}$ actually is measured from the statistics of number-counting of galaxies or pair-counting of galaxies. That is, the spatial distribution of the peculiar velocity of galaxies or cosmic matter, $\mathbf{v}(\mathbf{x})$, is not treated as a random field.

Recently, we developed the method of measuring the galaxy power spectrum with a space-scale (multiresolution) decomposition, i.e. measuring power spectrum in the representation of discrete wavelet transform (DWT) (Fang \& Feng 2000, Yang et al. 2001). Unlike eq.(1), the redshift distortion of the DWT power spectrum must be described by the statistics of the velocity field 
$\mathbf{v}(\mathbf{x})$, as the number-counting or pair-counting statistics are not enough. For instance, the redshift distortion of the power of a DWT mode $\psi(\mathbf{x})$ is dependent on the projection of the velocity field on this mode, i.e. $\int \mathbf{v}(\mathbf{x}) \psi(\mathbf{x}) d \mathbf{x}$, which cannot be simply measured by number-counting or pair-counting of galaxies.

The purpose of this paper is to develop the method of analyzing the velocity field of galaxies of dark matter particles as a random field. That is, we introduce the variables of the velocity field by an orthogonal DWT decomposition of the field, and calculate all statistics of velocity field by average over the ensemble of the field variables. With this field description, we study the problems referring eq.(1): 1.) the scale dependence of velocity dispersion (VD) and pairwise velocity dispersion (PVD); 2.) the PDF of peculiar velocity and pairwise velocity; 3.$)$ the local correlation between the velocity field and density field of cosmic matter.

Other motivation of studying velocity field is from the intermittency of cosmic density field. The analysis on high resolution data of QSO's Ly $\alpha$ forests has revealed that the cosmic mass field is significantly intermittent (Jamkhedkar, Zhan \& Fang 2000; Feng, Pando \& Fang 2001; Zhan, Jamkhedkar \& Fang 2001). That is, the PDF of local density fluctuations on small scales is neither gaussian nor exponential, but long-tailed. Taking into account the coupling between the local density fluctuations and peculiar velocity, we can expect that the PDF of the velocity field should be neither gaussian nor exponential, but long-tailed on small scales. This study also requires a decomposition by orthogonal basis, as a superposition of independent variables may erase the long-tail feature due to the central limit theorem.

The paper will be organized as follows. $§ 2$ introduces the multiresolution analysis of the velocity field. It will focus on the DWT description of the velocity dispersion (VD) and pairwise velocity dispersion (PVD). $§ 3$ develops the method of analyzing the scale-dependence of the VD and PVD, and demonstrate it with an uniformly random sample. $\S 4$ studies the VD and PVD of N-body simulated samples, including the scale dependence of the VD and PVD, the VD-to-PVD ratio, and the PDFs of the peculiar velocity and pairwise velocity. In $\S 5$, we show the correlation between the VD, PVD and the local density and local density fluctuations. Finally, the conclusions 
and discussions will be in $\S 6$.

\section{Velocity fields in the DWT representation}

\subsection{Peculiar velocity dispersion and relative velocity dispersion}

For a given galaxy catalog, one can write down a number density distribution of galaxies as

$$
n(\mathbf{x})=\sum_{n=1}^{N_{g}} w_{n} \delta^{D}\left(\mathbf{x}-\mathbf{x}_{n}\right),
$$

where $N_{g}$ is the total number of galaxies in the catalog, $\mathbf{x}_{n}$ is the position of the $n$th galaxy, $w_{n}$ is its weight, and $\delta^{D}$ the 3-D Dirac $\delta$ function. $n(\mathbf{x})$ is a sampling of the cosmic mass density distribution.

The velocity field, $\mathbf{v}(\mathbf{x})$, describes the deviation of particle motion from Hubble flow. The peculiar velocity of galaxies, $\mathbf{v}\left(\mathbf{x}_{n}\right)$ and $n=1 \ldots N_{g}$, is a sampling of the velocity field. Thus the continuous velocity field $\mathbf{v}(\mathbf{x})$ can be constructed by the convolution of the particle velocity with an assignment function $W(\eta)$,

$$
\mathbf{v}_{r}(\mathbf{x})=\frac{\sum_{n=1}^{N_{g}} W\left(\left|\mathbf{x}_{\mathbf{n}}-\mathbf{x}\right| / r\right) w_{n} \mathbf{v}\left(\mathbf{x}_{n}\right)}{\sum_{n=1}^{N_{g}} W\left(\left|\mathbf{x}_{\mathbf{n}}-\mathbf{x}\right| / r\right) w_{n}}
$$

where $r$ is a filter scale. One of the simplest example of assignment functions is the top-hat window function, i.e. $W(\eta)=1$ for $\eta \leq 1 / 2$ and 0 for $\eta>1 / 2$. Thus, $\mathbf{v}_{r}(\mathbf{x})$ can be considered as a sampling of the cosmic velocity field $\mathbf{v}(\mathbf{x})$ smoothed on the scale $r$.

Using $\mathbf{v}_{r}(\mathbf{x})$, the velocity dispersion (VD) is defined as the variance of the one-point distribution of the random variable $\mathbf{v}_{r}(\mathbf{x})$, i.e.

$$
\left[\sigma_{r}^{v}(\mathbf{x})\right]^{2}=\left\langle\mathbf{v}_{r}^{2}(\mathbf{x})\right\rangle
$$

where $\langle\ldots\rangle$ means the ensemble average.

Using eq.(3), one can define the relative velocity by

$$
\Delta \mathbf{v}_{r}(\mathbf{x})=\mathbf{v}_{r / 2}[\mathbf{x}+(\mathbf{r} / \mathbf{2})]-\mathbf{v}_{r / 2}[\mathbf{x}-(\mathbf{r} / \mathbf{2})]
$$


where $|\mathbf{r}|=r$. Obviously, $\Delta \mathbf{v}_{r}(\mathbf{x})$ measures the difference of the velocity field at $\mathbf{x}+\mathbf{r} / \mathbf{2}$ and $\mathbf{x}-\mathbf{r} / \mathbf{2}$. If $r$ is small, and there is only one galaxy $\mathbf{x}_{\mathbf{n}}$ in the sphere $r / 2$ at $\mathbf{x}+(\mathbf{r} / \mathbf{2})$, and one galaxy $\mathbf{x}_{\mathbf{m}}$ in the sphere $r / 2$ at $\mathbf{x}-(\mathbf{r} / \mathbf{2})$, we have $\Delta \mathbf{v}_{r}(\mathbf{x})=\mathbf{v}\left(\mathbf{x}_{\mathbf{n}}\right)-\mathbf{v}\left(\mathbf{x}_{\mathbf{m}}\right)$. Therefore, the measure eq.(5) contains all information of the relative velocity of individual galaxy pairs.

Yet, the relative velocity defined by eq.(5) is not completely the same as pairwise velocity of galaxies. Usually, the pairwise velocity is defined as $v_{12}-\overline{v_{12}}$, where $v_{12}$ is the relative velocity of galaxy pair 1 and 2 along line-of-sight, and $\overline{v_{12}}$ is the mean relative velocity, or the in-fall motion, which generally is modeled by a similarity solution (Davis \& Peebles 1983). Therefore, the measure of pairwise velocity is model-dependent.

The reason of using the relative velocity eq.(5) mainly is that the variables $\Delta \mathbf{v}_{r}(\mathbf{x})$ are able to provide an information-lossless and model-independent decomposition of the velocity field (§2.2). Moreover, the in-fall term of the pairwise velocity measure is negligible for small scales. Therefore, at least on small scales, the definition eq.(5) is a reasonable generalization of the pairwise velocity of galaxies on small scales. We may still call $\Delta \mathbf{v}_{r}(\mathbf{x})$ the variables of pairwise velocity decomposition.

Thus, the pairwise velocity dispersion (PVD) is defined as the variance of the one-point distribution of the random variable $\Delta \mathbf{v}_{r}(\mathbf{x})$, i.e.

$$
\left[\sigma_{r}^{p v}(\mathbf{x})\right]^{2}=\left\langle\left[\Delta \mathbf{v}_{r}(\mathbf{x})\right]^{2}\right\rangle
$$

\subsection{Need for scale-space decomposition}

A common feature of $\mathbf{v}_{r}(\mathbf{x})$ [eq.(3)] and $\Delta \mathbf{v}_{r}(\mathbf{x})$ [eq.(5)] is that each quantity depends on two variables: the position $\mathbf{x}$ and the scale $r$. That is, the VD and PVD measure the random velocity field under a space-scale decomposition. This point can be seen more clearly by rewriting eqs.(3) and (5) using eq.(2). We have

$$
\mathbf{v}_{r}(\mathbf{x})=\frac{\int n\left(\mathbf{x}^{\prime}\right) \mathbf{v}\left(\mathbf{x}^{\prime}\right) W\left(\left|\mathbf{x}^{\prime}-\mathbf{x}\right| / r\right) d \mathbf{x}^{\prime}}{\int n\left(\mathbf{x}^{\prime}\right) W\left(\left|\mathbf{x}^{\prime}-\mathbf{x}\right| / r\right) d \mathbf{x}^{\prime}}
$$


and

$$
\Delta \mathbf{v}_{r}(\mathbf{x})=\frac{\int n\left(\mathbf{x}^{\prime}\right) \mathbf{v}\left(\mathbf{x}^{\prime}\right) U_{r}\left(\mathbf{x}^{\prime}-\mathbf{x}\right) d \mathbf{x}^{\prime}}{\int n\left(\mathbf{x}^{\prime}\right) W\left(\left|\mathbf{x}^{\prime}-\mathbf{x}\right| / r\right) d \mathbf{x}^{\prime}}
$$

where function $U\left(\mathbf{x}^{\prime}-\mathbf{x}\right)$ is given by the subtractions of window functions, i.e.

$$
U_{r}\left(\mathbf{x}^{\prime}-\mathbf{x}\right)=W\left[\left|\mathbf{x}^{\prime}-\mathbf{x}+\mathbf{r} / \mathbf{2}\right| /(r / 2)\right]-W\left[\left|\mathbf{x}^{\prime}-\mathbf{x}-\mathbf{r} / \mathbf{2}\right| /(r / 2)\right]
$$

or

$$
U_{r}\left(\mathbf{x}^{\prime}-\mathbf{x}\right)= \begin{cases}1 & \left|\mathbf{x}^{\prime}-\mathbf{x}-\mathbf{r} / \mathbf{2}\right|<r / 2 \\ -1 & \left|\mathbf{x}^{\prime}-\mathbf{x}+\mathbf{r} / \mathbf{2}\right|<r / 2 \\ 0 & \text { otherwise. }\end{cases}
$$

Function $U_{r}\left(\mathbf{x}-\mathbf{x}^{\prime}\right)$ is similar to the Haar wavelet, of which in 1-D is

$$
\psi(x)= \begin{cases}1 & 0 \leq x \leq 1 / 2 \\ -1 & 1 / 2<x \leq 1 \\ 0 & \text { otherwise }\end{cases}
$$

Therefore, the pairwise velocity eq.(8) actually is a decomposition of the velocity field with a Haar wavelet-like function.

However, the functions $U_{r}\left(\mathbf{x}^{\prime}-\mathbf{x}\right)$ with respect to $\mathbf{x}$ and $r$ don't exhibit completeness and orthogonality. Therefore, the decomposition with $U_{r}\left(\mathbf{x}^{\prime}-\mathbf{x}\right)$ may lead to loss of information if $U$ 's are incomplete, or cause false correlations if they are redundant. To have a proper measure of the VD and PVD, we call on discrete wavelet transform (DWT), which provides a complete and unredundant space-scale decomposition. The first approach of describing cosmic velocity field by wavelet is given by Rauzy, Lachieze-Rey \& Henriksen (1993). However, they use continuous wavelets, which give rise to a redundant decomposition (Farge 1992).

\subsection{The DWT variables of velocity field}

For the details of the mathematical properties of the DWT refers to Mallat (1989a,b); Meyer (1992); Daubechies, (1992), and for physical applications, refer to Fang \& Thews (1998). The 
Haar wavelet [eq.(12)] provides a clear picture of the DWT decomposition, and it is also easy for numerical work. However, the Haar wavelet is discontinues, and therefore, it is not well localized in scale space. To our work, the most important properties of the basis for the scale-space decomposition are 1.) orthogonality, 2.) completeness, and 3.) locality in both scale and physical spaces. Therefore, wavelets with compactly supported basis are suitable to the velocity field analysis. Among the compactly supported orthogonal basis, the Daubechies 4 (D4) is easy for numerical calculation. We will use wavelet D4. The basic scaling function $\phi(x)$, basic wavelet $\psi(x)$ and their Fourier transform of the D4 are shown in Fig. 1.

To simplify the notation, we consider an 1-D density field $n(x)$ and velocity field $v(x)$ on spatial range $L$. The result is straightforward generalized to $3-\mathrm{D}$ fields in $§ 3.3$.

For doing the DWT analysis, the space $L$ is chopped into $2^{j}$ segments labelled by $l=0,1, \ldots 2^{j}-1$. Each of the segments has size $L / 2^{j}$. The index $j$ can be a positive integral. It stands for scale $L / 2^{j}$. The index $l$ is for position, and corresponds to spatial range $l L / 2^{j}<x<(l+1) L / 2^{j}$.

For a random field $n(x)$, the DWT analysis is performed by the scaling functions $\phi_{j, l}(x)=\left(2^{j} / L\right)^{1 / 2} \phi\left(2^{j} / L-l\right)$, and wavelets $\psi_{j, l}(x)=\left(2^{j} / L\right)^{1 / 2} \psi\left(2^{j} / L-l\right)$. The scaling functions play the role of window function. Generally, $\phi_{j, l}(x)$ is a window in the segment $l$. They are used to calculate the mean field in the segment $l$. The wavelets $\psi_{j, l}(x)$ essentially is similar to the function $U$ of eq.(10). They are used to extract the fluctuations of the fields at the segment $l$, i.e. they are used to calculate the difference between the mean fields at space ranges $l L / 2^{j}<x<(l+1 / 2) L / 2^{j}$ and $(l+1 / 2) L / 2^{j}<x<(l+1) L / 2^{j}$.

The scaling functions and wavelets $\psi_{j, l}(x)$ satisfy the orthogonal relations as

$$
\begin{gathered}
\int \phi_{j, l}(x) \phi_{j, l^{\prime}}(x) d x=\delta_{l, l^{\prime}}, \\
\int \psi_{j, l}(x) \psi_{j^{\prime}, l^{\prime}}(x) d x=\delta_{j, j^{\prime}} \delta_{l, l^{\prime}}, \\
\int \phi_{j, l}(x) \psi_{j^{\prime}, l^{\prime}}(x) d x=0, \quad \text { if } j^{\prime} \geq j .
\end{gathered}
$$


With these properties, a 1-D random field $n(x)$ can be decomposed into

$$
n(x)=n^{j}(x)+\sum_{j^{\prime}=j}^{\infty} \sum_{l=0}^{2^{j^{\prime}}-1} \tilde{\epsilon}_{j^{\prime}, l}^{n} \psi_{j^{\prime}, l}(x)
$$

where

$$
n^{j}(x)=\sum_{l=0}^{2^{j}-1} \epsilon_{j, l}^{n} \phi_{j, l}(x) .
$$

The scaling function coefficient (SFC) $\epsilon_{j, l}^{n}$ and the wavelet function coefficient (WFC), $\tilde{\epsilon}_{j, l}^{n}$ are given by

$$
\epsilon_{j, l}^{n}=\int n(x) \phi_{j, l}(x) d x
$$

and

$$
\tilde{\epsilon}_{j, l}^{n}=\int n(x) \psi_{j, l}(x) d x
$$

respectively. The SFC $\epsilon_{j, l}^{n}$ measures the mean $n(x)$ in the segment $l$, while the WFC $\tilde{\epsilon}_{j, l}^{n}$ measures the fluctuations of field $n(x)$ at $l$ on scale $j$.

The first term on the r.h.s. eq.(15), $n^{j}(x)$, is the field $n(x)$ smoothed on the scale $j$, while the second term contains all information on scales $\geq j$. Because of the orthogonal relation eq.(14), the decomposition between the scales of $<j$ (first term) and $\geq j$ (second term) in eq.(15) is unambiguous. The value of $j$ in eq.(15) can be any integer, and therefore, a scale-by-scale decomposition becomes possible.

Since scale $r$, position $x$, and window function $W\left(\left|x^{\prime}-x\right| / r\right)$ of eq.(3) correspond to, respectively, $j, l$, and $\phi_{j, l}\left(x^{\prime}\right)$ of the DWT analysis, the DWT counterpart of eq.(3) (for 1-D) is

$$
v_{j, l}=\frac{\int v(x) n(x) \phi_{j, l}(x) d x}{\int n(x) \phi_{j, l}(x) d x}=\frac{\epsilon_{j, l}^{v}}{\epsilon_{j, l}^{n}}
$$

where $\epsilon_{j, l}^{v}$ and $\epsilon_{j, l}^{n}$ are respectively, the SFC of field $v(x) n(x)$ and $n(x)$, i.e.

$$
\epsilon_{j, l}^{v}=\int v(x) n(x) \phi_{j, l}(x) d x=\sum_{n=1}^{N_{g}} w_{n} v\left(x_{n}\right) \phi_{j, l}\left(x_{n}\right)
$$

and

$$
\epsilon_{j, l}^{n}=\int n(x) \phi_{j, l}(x) d x=\sum_{n=1}^{N_{g}} w_{n} \phi_{j, l}\left(x_{n}\right) .
$$


$v_{j, l}$ is the mean velocity in the spatial range $l L / 2^{j}<x<(l+1) L / 2^{j}$.

Similarly, $U_{r}$ corresponds to $\psi_{j, l}$, and therefore, the DWT counterpart of eq.(5) is

$$
\Delta v_{j, l}=\frac{\int v(x) n(x) \psi_{j, l}(x) d x}{\int n(x) \phi_{j, l}(x) d x}=\frac{\tilde{\epsilon}_{j, l}^{v}}{\epsilon_{j, l}^{n}},
$$

where $\tilde{\epsilon}_{j, l}^{v}$ is the WFC of field $v(x) n(x)$, i.e.

$$
\tilde{\epsilon}_{j, l}^{v}=\int v(x) n(x) \psi_{j, l}(x) d x=\sum_{n=1}^{N_{g}} w_{n} v\left(x_{n}\right) \psi_{j, l}\left(x_{n}\right) .
$$

$\Delta v_{j, l}$ is the difference between the mean velocities of spatial ranges $l L / 2^{j}<x<(l+1 / 2) L / 2^{j}$ and $(l+1 / 2) L / 2^{j}<x<(l+1) L / 2^{j}$.

$v_{j, l}$ and $\Delta v_{j, l}$ are the variables of the velocity field $v(x)$ in the DWT representation. These variables give a complete description of the field $v(x)$ without loss of information. The orthogonality of scaling functions and wavelets insure that the decomposition does not cause false correlation among these variables.

It has been pointed out that the galaxy pairwise velocity dispersion measured by conventional techniques, i.e. pair-counting statistics, is biased by densest regions, as the statistic actually is pair-weighted (Strauss, Ostriker \& Cen 1998.) In the language of the velocity field decomposition, the conventional method is redundant for modes at the dense regions. In the DWT decomposition, each mode $(j, l)$ corresponds to a cell in phase space (scale $L / 2^{j}$ and position $l$ ), and is described by one variable $v_{j, l}$ or $\Delta v_{j, l}$. That is, all modes are treated equal. Each degree of freedom of the velocity field is represented by one variable, regardless the number of galaxies in the cell. Therefore, the description of $v_{j, l}$ and $\Delta v_{j, l}$ is free from the bias of pair-weight.

\section{The VD and PVD spectrum of a velocity field}




\subsection{The VD and PVD spectrum}

With eqs.(19) and (22), the VD and PVD are given by ensemble averages of $v_{j, l}^{2}$ and $\Delta v_{j, l}^{2}$, i.e.

$$
\sigma_{j, l}^{v}=\left\langle v_{j, l}^{2}\right\rangle^{1 / 2}=\left\langle\left[\frac{\int v(x) n(x) \phi_{j, l}(x) d x}{\int n(x) \phi_{j, l}(x) d x}\right]^{2}\right\rangle^{1 / 2}
$$

and

$$
\sigma_{j, l}^{p v}=\left\langle\Delta v_{j, l}^{2}\right\rangle^{1 / 2}=\left\langle\left[\frac{\int v(x) n(x) \psi_{j, l}(x) d x}{\int n(x) \phi_{j, l}(x) d x}\right]^{2}\right\rangle^{1 / 2} .
$$

If the field is statistically uniform, $\sigma_{j, l}^{v}$ and $\sigma_{j, l}^{p v}$ are independent of $l$. One can define the VD and PVD on scale $j$ by $\sigma_{j}^{v} \equiv \sigma_{j, l}^{v}$, and $\sigma_{j}^{p v} \equiv \sigma_{j, l}^{p v}$, which are referred to as the VD and PVD spectrum of the velocity field, respectively.

If the "fair sample hypothesis" (Peebles 1980) holds, the ensemble average $\langle\ldots\rangle$ can be replaced by a spatial average over $l$. Eqs.(24) and (25) become

$$
\sigma_{j}^{v}=\left[\frac{1}{2^{j}} \sum_{l=0}^{2^{j}-1} v_{j, l}^{2}\right]^{1 / 2}=\left[\frac{1}{2^{j}} \sum_{l=0}^{2^{j}-1}\left(\frac{\epsilon_{j, l}^{v}}{\epsilon_{j, l}^{n}}\right)^{2}\right]^{1 / 2} .
$$

and

$$
\sigma_{j}^{p v}=\left[\frac{1}{2^{j}} \sum_{l=0}^{2^{j}-1} \Delta v_{j, l}^{2}\right]^{1 / 2}=\left[\frac{1}{2^{j}} \sum_{l=0}^{2^{j}-1}\left(\frac{\tilde{\epsilon}_{j, l}^{v}}{\epsilon_{j, l}^{n}}\right)^{2}\right]^{1 / 2} .
$$

Eqs.(26) and (27) show that the VD and PVD of a velocity field are given by the SFCs and WFCs of the DWT variables, respectively. For a non-gaussian random field $F(x)$, the statistical behaviors of the field quantity $F(x)$ and its spatial difference $F(x)-F(x+r)$ generally are different. The statistical behaviors of the VD and PVD are different from each other.

As a useful variant of eq.(24), we define a modified VD by

$$
\bar{\sigma}_{j}^{v}=\left[\frac{\left\langle\left[\int v(x) n(x) \phi_{j, l}(x) d x\right]^{2}\right\rangle}{\left\langle\left[\int n(x) \phi_{j, l}(x) d x\right]^{2}\right\rangle}\right]^{1 / 2} .
$$

Obviously, if the distribution $n(x)$ is randomly uniform, $\bar{\sigma}_{j}^{v}$ will be the same as $\sigma_{j}^{v}$. If velocity field is long-range correlated, i.e. $\left\langle v(x) v\left(x^{\prime}\right)\right\rangle \simeq$ const, and no correlation with $n(x)$, we have also $\bar{\sigma}_{j}^{v} \simeq \sigma_{j}^{v}$, even $n(x)$ is not uniform. 
Because $\left\langle\left[\int v(x) n(x) \phi_{j, l}(x) d x\right]^{2}\right\rangle=\left(1 / 2^{j}\right) \sum\left(\epsilon_{\mathbf{j}, 1}^{v}\right)^{2}$ and $\left\langle\left[\int n(x) \phi_{j, l}(x) d x\right]^{2}\right\rangle=\left(1 / 2^{j}\right) \sum\left(\epsilon_{\mathbf{j}, 1}^{n}\right)^{2}$, eq.(28) yields

$$
\bar{\sigma}_{j}^{v}=\left[\frac{\sum_{l=0}^{2^{j}-1}\left(\epsilon_{\mathbf{j}, 1}^{v}\right)^{2}}{\sum_{l=0}^{2^{j}-1}\left(\epsilon_{\mathbf{j}, 1}^{n}\right)^{2}}\right]^{1 / 2} .
$$

Similarly, a modified PVD can be defined by

$$
\bar{\sigma}_{j}^{p v}=\left[\frac{\sum_{l=0}^{2^{j}-1}\left(\tilde{\epsilon}_{\mathbf{j}, 1}^{v}\right)^{2}}{\sum_{l=0}^{2^{j}-1}\left(\epsilon_{\mathbf{j}, 1}^{n}\right)^{2}}\right]^{1 / 2} .
$$

The difference between $\sigma_{j}^{v}$ and $\bar{\sigma}_{j}^{v}$, or $\sigma_{j}^{p v}$ and $\bar{\sigma}_{j}^{p v}$ can be used as an indicator of the correlations between velocity and density fields $(\S 5)$.

\subsection{An example: random fields}

To demonstrate the VD and PVD spectra, let's consider 1-D random fields, which are produced by uniformly random distribution of $N$ particles in range $L$. Each particle $n$ is assigned a velocity $v_{n}$ drawn from a Gaussian distribution of the velocity with zero mean and variance 300 $\mathrm{km} \mathrm{s}^{-1}$. The two-point correlation function of number density $\left\langle n(x) n\left(x^{\prime}\right)\right\rangle=\bar{n}^{2}=(N / L)^{2}$. The two-point velocity correlation function of velocity field $\xi^{v}\left(x-x^{\prime}\right) \equiv\left\langle v(x) v\left(x^{\prime}\right)\right\rangle$ would be

$$
\xi^{v}\left(x-x^{\prime}\right)=\left\{\begin{array}{lll}
(300)^{2} & \mathrm{~km}^{2} \mathrm{~s}^{-2} & \left|x-x^{\prime}\right| \simeq L / N \\
0 & & \left|x-x^{\prime}\right|>L / N .
\end{array}\right.
$$

In this case, $\sigma_{j}^{v} \simeq \bar{\sigma}_{j}^{v}$. Figure $1(\mathrm{a})$ displays the modified VD spectrum $\bar{\sigma}_{j}^{v}$ of the random samples with $L=1$ and $N=10^{3}, 10^{4}$ and $10^{5}$. Fig. 1(a) shows that $\bar{\sigma}_{j}^{v}$ increases with $j$, and saturates at $300 \mathrm{~km} / \mathrm{s}$. This behavior can be deduced analytically. In fact, following eq.(28) and $\left\langle n(x) n\left(x^{\prime}\right)\right\rangle=$ const, we have

$$
\begin{aligned}
\left(\bar{\sigma}_{j}^{v}\right)^{2} & =\frac{\iint\left\langle n(x) n\left(x^{\prime}\right)\right\rangle\left\langle v(x) v\left(x^{\prime}\right)\right\rangle \phi_{j, l}(x) \phi_{j, l}\left(x^{\prime}\right) d x d x^{\prime}}{\iint\left\langle n(x) n\left(x^{\prime}\right)\right\rangle \phi_{j, l}(x) \phi_{j, l}\left(x^{\prime}\right) d x d x^{\prime}} \\
& =\frac{\iint \xi^{v}\left(x-x^{\prime}\right) \phi_{j, l}(x) \phi_{j, l}\left(x^{\prime}\right) d x d x^{\prime}}{\iint \phi_{j, l}(x) \phi_{j, l}\left(x^{\prime}\right) d x d x^{\prime}}
\end{aligned}
$$

Considering that both $\phi_{j, l}(x)$ and $\phi_{j, l}\left(x^{\prime}\right)$ are window-like functions in the spatial range $l L / 2^{j}<x, x^{\prime}<(l+1) L / 2^{j}$, the integral of the denominator of eq.(31) is proportional to area 
$L / 2^{j} \times L / 2^{j}$. On the other hand, the numerator is proportional to area $L / 2^{j} \times L / N$, the factor $L / N$ is due to the condition $\left|x-x^{\prime}\right| \simeq L / N$ in eq.(31). Thus, the VD of eq.(32) is

$$
\bar{\sigma}_{j}^{v} \simeq \begin{cases}2^{j / 2} 300 / \sqrt{N} \mathrm{~km}^{2} \mathrm{~s}^{-2} & \text { if } j \ll \ln _{2} N \\ 300 \mathrm{~km}^{2} \mathrm{~s}^{-2} & \text { if } j \simeq \ln _{2} N\end{cases}
$$

This is just what as indicated in Fig. 1(a).

Figure 1(b) gives the modified PVD spectrum calculated by eq.(30). It shows the same as the VD spectrum, i.e. $\bar{\sigma}_{j}^{p v}=\bar{\sigma}_{j}^{v}$. This is expected. For the VD eq.(29), the SFC $\epsilon_{j, l}^{v}$ is given by the addition of the velocities of galaxies within the cell $(j, l)$, while for the PVD eq.(30), the WFC $\tilde{\epsilon}_{j, l}^{v}$ is given by the difference between the velocities of galaxies in $L l / 2^{j+1}$ and $L(l+1 / 2) / 2^{j+1}$. However, the one point function of the velocity is Gaussian, the probability of positive and negative velocity is the same, and therefore, the statistical properties of the SFC and WFC are the same. Consequently, the VD and PVD spectra coincide for this random field.

From Figs. 1(a) and 1(b), we can conclude that if the velocities of galaxies are uncorrelated on scales larger than $j$, the VD and PVD spectra will decrease systematically with the $j$ decreasing by a law $2^{j / 2}(1-\mathrm{D})$. This result is useful to estimate the scale of the correlation length of velocity field of galaxies.

\subsection{3-D velocity field}

For 3-D velocity field $\mathbf{v}(\mathbf{x}), \mathbf{x}=\left(x^{1}, x^{2}, x^{3}\right)$, the DWT decomposition is based on the 3 -D scaling functions and wavelets, which can be constructed by a direct product of 1-D wavelet basis (Fang \& Thews 1998). For instance, in a 3 -D volume $L_{1} \times L_{2} \times L_{3}$, we have scaling function as

$$
\phi_{\mathbf{j}, 1}(\mathbf{x})=\phi_{j_{1}, l_{1}}\left(x_{1}\right) \phi_{j_{2}, l_{2}}\left(x_{2}\right) \phi_{j_{3}, l_{3}}\left(x_{3}\right)
$$

and wavelets as

$$
\psi_{\mathbf{j}, 1}(\mathbf{x})=\psi_{j_{1}, l_{1}}\left(x_{1}\right) \psi_{j_{2}, l_{2}}\left(x_{2}\right) \psi_{j_{3}, l_{3}}\left(x_{3}\right)
$$

where $\mathbf{j}=\left(j_{1}, j_{2}, j_{3}\right), \mathbf{l}=\left(l_{1}, l_{2}, l_{3}\right)$, and $l_{i}=0 \ldots 2^{j_{i}-1}, i=1,2,3$. The wavelet $\psi_{\mathbf{j}, 1}(\mathbf{x})$ is non-zero mainly in a volume $L_{1} / 2^{j_{1}} \times L_{2} / 2^{j_{2}} \times L_{3} / 2^{j_{3}}$, and around the position 
$\left(x_{1}=l_{1} L_{1} / 2^{j_{1}}, x_{2}=l_{2} L_{2} / 2^{j_{2}}, x_{3}=l_{3} L_{3} / 2^{j_{3}}\right)$, i.e. they localized in both scale and physical space.

The 3-D generalization of the DWT variables $v_{j, l}$ [eq.(19)] and $\Delta v_{j, l}$ [eq.(22)] are given by

$$
v_{i} \mathbf{j}, \mathbf{l}=\frac{\epsilon_{\mathbf{j}, \mathbf{l}}^{v_{i}}}{\epsilon_{\mathbf{j}, \mathbf{l}}^{n}}
$$

and

$$
\Delta v_{i \mathbf{j}, 1}=\frac{\tilde{\epsilon}_{\mathbf{j}, 1}^{v_{i}}}{\epsilon_{\mathbf{j}, 1}^{n}}
$$

where $\epsilon_{\mathbf{j}, 1}^{v_{i}}$ and $\epsilon_{\mathbf{j}, 1}^{n}$ are the SFCs as

$$
\begin{gathered}
\epsilon_{\mathbf{j}, \mathbf{l}}^{v_{i}}=\int n(\mathbf{x}) v_{i}(\mathbf{x}) \phi_{\mathbf{j}, \mathbf{l}}(\mathbf{x}) d \mathbf{x}=\sum_{n=1}^{N_{g}} w_{n} v_{i}\left(\mathbf{x}_{n}\right) \phi_{\mathbf{j}, 1}\left(\mathbf{x}_{n}\right), \\
\epsilon_{\mathbf{j}, \mathbf{l}}^{n}=\int n(\mathbf{x}) \phi_{\mathbf{j}, \mathbf{l}}(\mathbf{x}) d \mathbf{x}=\sum_{n=1}^{N_{g}} w_{n} \phi_{\mathbf{j}, \mathbf{l}}\left(\mathbf{x}_{n}\right)
\end{gathered}
$$

and $\tilde{\epsilon}_{\mathbf{j}, 1}^{v_{i}}$ is the WFC as

$$
\tilde{\epsilon}_{\mathbf{j}, 1}^{v_{i}}=\int v_{i}(\mathbf{x}) \psi_{\mathbf{j}, 1}(\mathbf{x}) d \mathbf{x}=\sum_{n=1}^{N_{g}} w_{n} v_{i}\left(\mathbf{x}_{n}\right) \psi_{\mathbf{j}, 1}\left(\mathbf{x}_{n}\right) .
$$

The SFCs $\epsilon_{\mathbf{j}, 1}^{n}$ [eq.(39)] are assigned at regular grids $l_{i}=0 \ldots 2^{j_{i}}$ and $i=1,2,3$. That is, $\epsilon_{\mathbf{j}, 1}^{n}$ is an assignment of the number density distribution $n(\mathbf{x})$ onto grids $(\mathbf{j}, \mathbf{l})$. Eq.(39) shows that the $n$th galaxy (or particle) is assigned onto grid $\mathbf{j}, \mathbf{l}$ by weight $\phi_{\mathbf{j}, \mathbf{l}}\left(\mathbf{x}_{n}\right)$ (Fang \& Feng 2000). Similarly, SFCs $\epsilon_{\mathbf{j}, 1}^{v_{i}}$ is an assignment of the distribution $v_{i}(\mathbf{v}) n(\mathbf{x})$ onto grid $\mathbf{j}, \mathbf{l}$. Therefore, eqs.(36) and (37) essentially are the same as the DWT mass assignment (Fang \& Feng, 2000). We may call it the DWT velocity assignment.

From eqs.(36) and (37), we have

$$
\begin{gathered}
\sigma_{\mathbf{j}}^{v_{i}}=\left[\left\langle\left(v_{i} \mathbf{j}, \mathbf{l}\right)^{2}\right\rangle\right]^{1 / 2}=\left[\frac{1}{2^{\left(j_{1}+j_{2}+j_{3}\right)}} \sum_{\mathbf{l}=0}^{2^{\mathbf{j}}-1}\left(\frac{\epsilon_{\mathbf{j}, \mathbf{l}}^{v_{i}}}{\epsilon_{\mathbf{j}, \mathbf{l}}^{n}}\right)^{2}\right]^{1 / 2}, \\
\bar{\sigma}_{\mathbf{j}}^{v_{i}}=\left[\frac{\sum_{\mathbf{l}=0}^{2^{\mathbf{j}}-1}\left(\epsilon_{\mathbf{j}, \mathbf{l}}^{v_{i}}\right)^{2}}{\sum_{\mathbf{l}=0}^{2^{\mathbf{j}}-1}\left(\epsilon_{\mathbf{j}, \mathbf{l}}^{n}\right)^{2}}\right]^{1 / 2}
\end{gathered}
$$

for 3-D VD and modified VD, and

$$
\sigma_{\mathbf{j}}^{p v_{i}}=\left[\left\langle\left(\Delta v_{i} \mathbf{j}, \mathbf{l}\right)^{2}\right\rangle\right]^{1 / 2}=\left[\frac{1}{2^{\left(j_{1}+j_{2}+j_{3}\right)}} \sum_{\mathbf{l}=\mathbf{0}}^{2^{\mathbf{j}}-1}\left(\frac{\tilde{\epsilon}_{\mathbf{j}, \mathbf{l}}^{v_{i}}}{\epsilon_{\mathbf{j}, \mathbf{l}}^{n}}\right)^{2}\right]^{1 / 2},
$$




$$
\bar{\sigma}_{\mathbf{j}}^{p v_{i}}=\left[\frac{\sum_{\mathbf{l}=\mathbf{0}}^{2^{\mathbf{j}}-1}\left(\tilde{\epsilon}_{\mathbf{j}, \mathbf{l}}^{v_{i}}\right)^{2}}{\sum_{\mathbf{l}=\mathbf{0}}^{2^{\mathbf{j}}-1}\left(\epsilon_{\mathbf{j}, \mathbf{l}}^{n}\right)^{2}}\right]^{1 / 2}
$$

for 3-D PVD and modified PVD.

\section{Velocity field of simulation samples}

\subsection{Simulation samples}

To demonstrate the velocity field of cosmic matter, we produce ten realizations of the N-body simulation for each model of the SCDM, $\tau \mathrm{CDM}$ and $\Lambda \mathrm{CDM}$. The parameters $\left(\Omega_{0}, \Lambda, \Gamma, \sigma_{8}\right)$ are taken to be $(1.0,0.0,0.5,0.55)$ for standard CDM model (SCDM), (1.0,0.0,0.25,0.55) for a variant of SCDM model $(\tau \mathrm{CDM})$ and $(0.3,0.7,0.21,0.85)$ for a low density flat model $(\Lambda \mathrm{CDM})$. We use modified $\mathrm{AP}^{3} \mathrm{M}$ code (Couchman, 1991) to evolve $128^{3}$ cold dark matter particles in a periodic cube of side length $\mathrm{L}=256 \mathrm{~h}^{-1} \mathrm{Mpc}$. The linear power spectrum is taken from the fitting formula given in Bardeen et.al. (1986).

In our simulation, the so-called "glass" configuration is used to generate the unperturbed uniform distribution of particles, and the Zel'dovich approximation is then applied to set up the initial perturbation. For the mass assignment on the grid and the calculation of the force on a given particle from interpolation of the grid values, we use the triangular-shaped cloud (TSC) method. The starting redshift of the simulation is taken to be $z_{i}=15$ for the SCDM model and $z_{i}=25$ for $\Lambda \mathrm{CDM}$ and $\tau \mathrm{CDM}$. The force softening parameter $\eta$ in the comoving system decreases with time as $\eta \propto 1 / a(t)$. Its initial value is taken to be $\eta=384 \mathrm{~h}^{-1} \mathrm{kpc}$ and the minimum value

to be $\eta_{\min }=128 \mathrm{~h}^{-1} \mathrm{kpc}$, corresponding to $15 \%$ and $5 \%$ of the grid size respectively. We use the "leap-frog" scheme in the single-step integration of time evolution, and take 600 total integration steps down to $z=0$. 


\subsection{The VD and PVD spectra of the simulation samples}

Now we calculate the VD spectrum of the simulation samples by eqs.(41) and (42), and the PVD spectrum by eqs.(43) and (44). A problem in using eqs.(41) and (43) is the ambiguity caused by extremely low values of galaxy number density in voids or underdense regions in the galaxy distribution, i.e. $n(\mathbf{x}) \simeq 0$. Within these areas, the corresponding SFCs $\epsilon_{\mathbf{j}, 1}^{n}$ would be very small, even zero, and hence, lead to very large terms of $\epsilon_{\mathbf{j}, 1}^{v_{i}} / \epsilon_{\mathbf{j}, 1}^{n}$. These terms are unacceptable, because in these areas the number density $n(\mathbf{x})$ actually are dominated by Poisson fluctuations. To control these terms, we use the so-called "off-counting" algorithm, of which the details are given in Appendix.

Since the cosmic velocity field is isotropic, the VD or PVD for the three components of $v^{i}$ and $p v^{i} i=1,2,3$ should be the same, i.e.

$$
\sigma_{\mathbf{j}}^{v_{1}}=\sigma_{\mathbf{j}}^{v_{2}}=\sigma_{\mathbf{j}}^{v_{3}}, \quad \sigma_{\mathbf{j}}^{p v_{1}}=\sigma_{\mathbf{j}}^{p v_{2}}=\sigma_{\mathbf{j}}^{p v_{3}} .
$$

Therefore, it is enough to calculate one component among $v_{i}$ and $p v_{i}$.

We consider diagonal mode, i.e. $j_{1}=j_{2}=j_{3}=j$. In this case, the VD, $\sigma_{j}^{v_{i}}$, and PVD, $\sigma_{j}^{p v_{i}}$, depend only on one scale parameter $j$. The physical scale of mode $j$ is $256 / 2^{j} \mathrm{~h}^{-1} \mathrm{Mpc}$, or wavenumber $k=2^{j+1} \sqrt{3} \pi / 256$. Figures 3 and 4 display, respectively, the $j$ - or $k$-dependence of $\sigma_{j}^{v_{i}}$ and $\bar{\sigma}_{j}^{v_{i}}$ for models of SCDM, $\tau \mathrm{CDM}$ and $\Lambda \mathrm{CDM}$. In comparison, the figures also plot the VD spectra for two types of random samples, which are given by assigning $N_{g}$ velocities to randomly distributed particles (hereafter random1), and randomizing the assignment of the $N_{g}$ velocities with particles $\mathbf{x}_{n}\left(n=1 \ldots N_{g}\right)$ (hereafter random2).

Figures 5 and 6 give, respectively, the $j$ - or $k$-dependence of $\sigma_{j}^{p v_{i}}$ and $\bar{\sigma}_{j}^{p v_{i}}$ of the simulation and random samples for models of SCDM, $\tau$ CDM and $\Lambda$ CDM.

As mentioned in $\S 3.2$, if the peculiar velocities are not correlated on scales larger than $j$, or $\xi^{v}\left(\mathbf{x}-\mathbf{x}^{\prime}\right) \simeq 0$ for $\left|\mathbf{x}-\mathbf{x}^{\prime}\right|>L / 2^{j}$, the VD and PVD spectra will decrease with the decreasing $j$ according to the law of $2^{j / 2}$ (1-D) [eq.(33)], or $2^{3 j / 2}(3-\mathrm{D})$. The spectra of random samples of Figs. 3 and 5 are decreasing with $j$ as the $2^{3 j / 2}$ law. However, the VD spectra of the simulation samples 
are almost flat, i.e. scale-independent, in the range of $k>0.2 \mathrm{~h} \mathrm{Mpc}^{-1}$, and slightly decreasing for $k<0.2 \mathrm{~h} \mathrm{Mpc}^{-1}$. This result shows that the peculiar velocities are spatially correlated on scales at least few $10 \mathrm{~h}^{-1} \mathrm{Mpc}$. This is probably caused by the in-fall process of forming halos on scales 10 and $20 \mathrm{~h}^{-1} \mathrm{Mpc}(\mathrm{Xu}$, Fang \& Wu 2000.)

The PVD spectrum decreases with $j$ on scale $k \simeq 1 \mathrm{~h} \mathrm{Mpc}^{-1}$, but it is slower than the $2^{3 j / 2}$ law. Accordingly, the correlation of the PVD on large scales is also significant.

Recall that in the recovery of the real space Fourier power spectrum from galaxy redshift surveys, to account for the redshift distortion effect, it is usually assumed that the peculiar velocity dispersion $\sigma^{v}$ is scale-independent. The DWT analysis show that the assumption of a constant $\sigma^{v}$ is reasonable on scales $k>0.2 \mathrm{~h} \mathrm{Mpc}^{-1}$, but may have uncertainty of factor 2 or 3 on large scales.

Although the VD and PVD of CDM models have similar scale-dependence, a difference between the VD and PVD can already be seen from Figs. 3 - 6. Figs. 3 and 4 show that for the CDM models, the modified VD $\bar{\sigma}_{j}^{v_{i}}$ is almost the same as $\sigma_{j}^{v_{i}}$ on all scales, while their $\sigma_{j}^{p v_{i}}$ are generally larger than $\bar{\sigma}_{j}^{p v_{i}}$, especially on small scales (Figs. 5 and 6). This result indicates that the peculiar velocity possesses long self-correlation length, but is less correlated with local number density.

This conclusion can also be seen from the behavior of random 1 and random 2 samples. The only difference between random 1 and random 2 is that the former has a uniform distribution $n(\mathbf{x})$ while the latter is inhomogeneous. Figs. 3 - 4 shows that for random1 the modified VD is the same as the VD, but for random2, the modified VD is substantially lower than the VD on small scales. The latter is due to the clustering of density field $n(\mathbf{x})$. The summation $\frac{1}{2^{\mathbf{j}}} \sum_{\mathbf{l}=0}^{2^{\mathbf{j}}-1}\left[1 / \epsilon_{\mathbf{j}, 1}^{n}\right]^{2}$ general is larger than $1 /\left(\frac{1}{2^{\mathbf{j}}} \sum_{\mathbf{l}=0}^{2^{\mathbf{j}}-1}\left[\epsilon_{\mathbf{j}, 1}^{n}\right]^{2}\right)$ for inhomogeneous distribution $n(\mathbf{x})$. Thus, the result of $\bar{\sigma}_{j}^{v_{i}} \simeq \sigma_{j}^{v_{i}}$ for CDM models means that the correlation of peculiar velocity field is not affected by the inhomogeneity density field. 


\subsection{The VD-to-PVD ratio (Mach number)}

The difference between the VD and PVD can be measured by the VD-to-PVD ratio as

$$
M_{j}=\frac{\sigma_{j}^{v_{i}}}{\sigma_{j}^{p v_{i}}} .
$$

This ratio is essentially to measure the relative strength of the mean velocity and the fluctuations of velocity field on scale $j$, i.e. the ratio between the bulk velocity and velocity fluctuations. Therefore, it is similar to the so-called Mach number (e.g. Suto \& Fujita 1990).

For the randomized samples, the VD and PVD spectra are identical (§3.2), as both spectra follow the $2^{3 j / 2}$ (3-D) law. Upon the definition of eq.(46), the Mach numbers of the random samples of $\S 3.2$ are equal to 1 on all scales. For simulation samples, the VD spectrum is no longer the same as PVD spectrum on all scales. The deviation of $M_{j}$ from one is a measure of evolved velocity field. Generally, $\sigma_{j}^{v_{i}}$ is larger than $\sigma_{j}^{p v_{i}}$ on large scales, i.e. the bulk flow is significantly larger than the PVD. This is due to the in-fall evolution. But $\sigma_{j}^{v_{i}}$ might be is less than $\sigma_{j}^{p v_{i}}$ on small scales.

Figure 7 plots the Mach numbers on scales $j=1-7$ for the three dark matter models. The figure shows that $M_{j}$ has a peak at $k \simeq 0.2 \mathrm{~h} \mathrm{Mpc}^{-1}$ for SCDM and $\tau \mathrm{CDM}$, and $k \simeq 0.1$ for the $\Lambda$ CDM. The peak characterizes a typical scale on which the matter undergoes the in-fall evolution. The clustering in the $\Lambda$ CDM model is earlier than the other two models, and therefore, the $\Lambda$ CDM's peak is on larger scale than the two others. On scales much larger than the peak, the mass and velocity fields are only slightly different from a Gaussian field, the VD and PVD are of the same order. On small scales, $M_{j}$ becomes small, and finally $\leq 1$, i.e. the PVD is larger than the VD. This scale-dependence can clearly be seen with the $\Lambda$ CDM model.

It has to be pointed out that the Mach number $M_{j}$ is a statistical measure of the entire velocity field, rather for individual object. Since the VD and PVD have different distribution functions (§4.5), the Mach number measured from individual objects might be significantly different from the Mach number of the entire velocity field. 


\subsection{Comparing DWT method with conventional techniques}

It would be interesting to compare the DWT measurement of VD and PVD with conventional techniques. Usually, the bulk velocity is given by the amplitude of the $R_{s}$-smoothed velocity field $v_{i}(\mathbf{x})$ over a volume defined by a normalized window function $W_{R}(\mathbf{x})$ of a characteristic scale $R$

$$
V_{i}(R)=\int W_{R}(\mathbf{x}) v_{i}(\mathbf{x}) d \mathbf{x}
$$

We calculate the variance $\left\langle V_{i}^{2}(R)\right\rangle^{1 / 2}$ with gaussian window function $W_{R} \propto \exp \left(-r^{2} / 2 R_{s}^{2}\right)$ for SCDM simulation sample. The result is shown in Fig. 8. We also plot $\sigma_{j}^{v_{i}}$ (SCDM) in Fig. 8. The data $\sigma_{j}^{v_{i}}(\mathrm{SCDM})$ is the same as Fig. 3. Since the effective radius of the gaussian window is $R=(3 \sqrt{2 \pi})^{1 / 3} R_{s}$, it corresponds to $j$ given by $R=(3 / 4 \pi)^{1 / 3} 256 / 2^{j} \mathrm{~h}^{-1} \mathrm{Mpc}$.

Figure 8 shows that the DWT gives exactly the same variance of bulk velocity as that measured by gaussian smooth.

To compare with the PDV of Fig. 5, we calculate the pairwise velocity of particles, $v_{12}(r=|\mathbf{r}|)=|\hat{r} \cdot[\mathbf{v}(\mathbf{x}+\mathbf{r})-\mathbf{v}(\mathbf{x})]|$, by pair-counting method. The variance of pairwise velocity, $\left\langle v_{12}^{2}(r)\right\rangle^{1 / 2}$, for the SCDM sample is shown in Fig. 9. The data of $\sigma_{j}^{p v_{i}}$ of Fig. 5 is also shown in Fig. 9. It is interested to see that scales less than $5 \mathrm{~h}^{-1} \mathrm{Mpc}$, we have $\left\langle v_{12}^{2}(r)\right\rangle^{1 / 2} \simeq \sqrt{3} \sigma_{j}^{p v_{i}}$ and $\left\langle V_{12}^{2}(R)\right\rangle^{1 / 2} \simeq \sqrt{2} \sqrt{3}\left\langle V_{i}^{2}(R=r)\right\rangle^{1 / 2}$. This result implies that velocity distribution on these scales is virialized, or quasi-virialized (Xu, Fang \& Wu 2000).

On scales larger than $5 \mathrm{~h}^{-1} \mathrm{Mpc},\left\langle v_{12}^{2}(r)\right\rangle^{1 / 2}$ generally is larger than $\sqrt{3} \sigma_{j}^{p v_{i}}$, but less than $\sqrt{2} \sqrt{3}\left\langle V_{i}^{2}(R)\right\rangle^{1 / 2}$. The large value of $\left\langle v_{12}^{2}(r)\right\rangle^{1 / 2}$ probably is because pair-counting measurement is not orthogonal with the $V_{i}(R)$ measurement, i.e. the pair-counting techniques may be biased by $\left\langle v_{12}^{2}(r)\right\rangle^{1 / 2}$.

The difference between field variable and conventional description of velocity field can easily be seen in linear regime of the cosmic clustering. It is well known that the linear relation between the density fluctuations and peculiar velocity field $\delta(\mathbf{x})=-\left(1 / H_{0} \beta\right) \nabla \cdot \mathbf{v}(\mathbf{x})$ yields

$$
\left\langle V^{2}(R)\right\rangle=\frac{H_{0}^{2} \beta^{2}}{2 \pi^{2}} \int_{0}^{\infty} d k P(k) \hat{W}_{R}^{2}(k),
$$


where $\left\langle V^{2}(R)\right\rangle=\sum_{i=1}^{3}\left\langle V_{i}^{2}(R)\right\rangle, \beta$ is the redshift distortion parameters, $P(k)$ the Fourier power spectrum of $\delta(\mathbf{x})$, and $\hat{W}_{R}(k)$ the Fourier transform of $W_{R}(\mathbf{x})$. In the DWT representation, the $\delta(\mathbf{x})-\mathbf{v}(\mathbf{x})$ linear relation gives

$$
\left[\sigma_{j}^{v}\right]^{2}=\frac{H_{0}^{2} \beta^{2}}{(2 \pi)^{2} L} \sum_{n_{1}, n_{2}, n_{3}=-\infty}^{\infty} \frac{1}{n_{1}^{2}+n_{2}^{2}+n_{3}^{2}} P(n)\left|\hat{\phi}\left(n_{1} / 2^{j}\right) \hat{\phi}\left(n_{2} / 2^{j}\right) \hat{\phi}\left(n_{3} / 2^{j}\right)\right|^{2},
$$

where $\left[\sigma_{j}^{v}\right]^{2}=\sum_{i=1}^{3}\left[\sigma_{j}^{v_{i}}\right]^{2}$, the Fourier power spectrum $P(n)$ is a function of $n$ related to the wavenumber by $k=2 \pi n / L, n^{2}=n_{1}^{2}+n_{2}^{2}+n_{3}^{2}$, and $\hat{\phi}$ is the Fourier transform of the basic scaling function $\phi(n)$ (Fig. 1).

Therefore, the only difference of the DWT measurement of VD from conventional method is to replace the ordinary window function $\hat{W}_{R}^{2}(k)$ by the DWT window $\left|\hat{\phi}\left(n_{1} / 2^{j}\right) \hat{\phi}\left(n_{2} / 2^{j}\right) \hat{\phi}\left(n_{3} / 2^{j}\right)\right|^{2}$. Fig.1 shows that $|\hat{\phi}(n)|^{2}$ is similar to ordinary window. This is why $\left\langle V^{2}(R)\right\rangle \simeq\left[\sigma_{j}^{v}\right]^{2}$ if the windows have the same size.

With the linear relation between $\delta(\mathbf{x})$ and $\mathbf{v}(\mathbf{x})$, the velocity dispersion within a patch of the velocity field sometimes is described by

$$
\left\langle\Delta V^{2}(R)\right\rangle=\frac{H_{0}^{2} \beta^{2}}{2 \pi^{2}} \int_{0}^{\infty} d k P(k)\left[1-\hat{W}_{R}^{2}(k)\right]
$$

The DWT counterpart of eq.(50) is given by the pairwise velocity. It is

$$
\left[\sigma_{j}^{p v}\right]^{2}=\frac{H_{0}^{2} \beta^{2}}{(2 \pi)^{2} L} \sum_{n_{1}, n_{2}, n_{3}=-\infty}^{\infty} \frac{1}{n_{1}^{2}+n_{2}^{2}+n_{3}^{2}} P(n)\left|\hat{\psi}\left(n_{1} / 2^{j}\right) \hat{\psi}\left(n_{2} / 2^{j}\right) \hat{\psi}\left(n_{3} / 2^{j}\right)\right|^{2},
$$

where $\left[\sigma_{j}^{p v}\right]^{2}=\sum_{i=1}^{3}\left[\sigma_{j}^{p v}\right]^{2}$, and $\hat{\psi}$ is the Fourier transform of basic wavelet $\psi$.

It is clearly to see the difference between eqs.(50) and (51). The r.h.s. of eq.(50) contains all contribution of $P(k)$ on scale $k R \geq 1$. On the other hand, Fig. 1 shows that $|\hat{\psi}(n)|^{2}$ is localized at $n \simeq \pm 1$, and therefore, the r.h.s. of eq.(51) contains only few terms around $n \simeq \sqrt{3} 2^{j}$. This shows that eq.(51) is based on unambiguous scale-decomposition.

As for the conventional pair-counting measurement of PVD, one cannot write down a linear relation between $\left\langle v_{12}^{2}(r)\right\rangle^{1 / 2}$ and $P(k)$ as eqs.(50) or (51). Although $r$ is used as a scale-indicator, the pair-counting method rely on a scale-decomposition of the distance $r$ of pairs, but not a scale 
decomposition of the field. Therefore, the statistics of $\left\langle V_{12}^{2}(R)\right\rangle^{1 / 2}$ are different from $\left[\sigma_{j}^{p v}\right]^{2}$ based on ensemble of the field variables.

Eqs.(49) and (51) shows also the difference between the VD, $\sigma_{j}^{v}$, and $\mathrm{PVD}, \sigma_{j}^{p v}$. The VD contains the contributions of $P(n)$ on all scales $n<2^{j}$. That is, even for a small scale $L / 2^{j}, \sigma_{j}^{v}$ is mainly determined by density perturbation on scales larger tha $L / 2^{j}$. The PVD, $\sigma_{j}^{p v}$, however is determined by $P(n)$ on the scales $L / 2^{j}$. From the DWT analysis, we have $|\hat{\psi}(n)|^{2}=|\hat{\phi}(n / 2)|^{2}-|\hat{\phi}(n)|^{2}$. Therefore, the PVD is determined by the power of density perturbations in the scale range from $L / 2^{j}$ to $L / 2^{j+1}$.

Generally, density perturbations on large scale is linear, while non-linear on small scales. Therefore, eq.(49) would be a good approximation for the VD, even on small scales, while eq.(51) would be a poor approximation for the PVD on scales for which the non-linear clustering is onset. This can be seen from Figs. 8 and 9. For the VD (Fig. 8), the theory [eq.(49)] and numerical simulation gives basically coincident results. However, for the PVD (Fig. 9), the result given by eq.(51) is significantly lower than numerical simulation. Therefore, one may conclude that the VD and PVD describes, respectively, the linear (large scales) and non-linear (small scales) behavior of the cosmic mass field.

\subsection{One-point distribution of $v_{i}$ and $\Delta v_{i}$}

The PDF of the galaxy pair velocity is usually modeled as an exponential, $f(\Delta v) \propto e^{-2^{1 / 2} \Delta v / \sigma^{p v}}$. This model is supported by best fitting of early galaxy redshift surveys, such as $14.5 m_{b}$ CfA (Davis \& Peebles 1993) and 1.2 Jy IRAS (Fisher et al. 1994.) Some non-linear clustering models also yield exponential distribution of pair velocity (Sheth 1996, Diaferio \& Geller, 1996). However, these results were measured by conventional techniques, it cannot directly be applied to variables $v_{i} \mathbf{j}, 1$ or $\Delta v_{i} \mathbf{j}, 1$. We calculate one-point distributions of field variables $v_{i}$ and $\Delta v_{i}$.

For a given scale $j$, the $2^{\left(j_{1}+j_{2}+j_{3}\right)}$ values of $v_{i} \mathbf{j}, \mathbf{l}\left(\right.$ or $\left.\epsilon_{\mathbf{j}, 1}^{v_{i}} / \epsilon_{\mathbf{j}, \mathbf{l}}\right)$ form an ensembles of $v_{i} \mathbf{j}, \mathbf{l}$. Thus, 
the one-point distributions $f\left(v_{i}\right)$ on scale $j$ can be obtained directly from the distribution of $v_{i} \mathbf{j}, 1$.

Figure 10 gives the one-point distribution $f\left(v_{i}\right)$ on scales $j=2-7$ of the SCDM. Models of $\tau \mathrm{CDM}$ and $\Lambda \mathrm{CDM}$ give the similar results. Figure 10 shows that the distributions are scale-dependent. On large scales $j=2,3$ the distribution is close to a Gaussian. On small scales $j=6,7$ the distribution in the middle range $\left(\left|v_{i}\right|<450 \mathrm{~km} / \mathrm{s}\right)$ can still be fitted with a Gaussian function. In the range $400<v_{i}<1200 \mathrm{~km} / \mathrm{s}, f\left(v_{i}\right)$, however, follows a straight line which implies that the distribution is exponential. With increasing $v_{i}>1200 \mathrm{~km} / \mathrm{s}, f\left(v_{i}\right)$ decays slower than a straight line. It indicates that the tail of the one-point distribution $f\left(v_{i}\right)$ extends further than an exponential distribution. Therefore, only in the middle velocity range, we can fit the distribution $f\left(v_{i}\right)$ by an exponential.

From Fig. 8 and eqs.(48) and (49), one can expect that the PDF of VD measured by a conventional Gaussian window should be about the same as Fig. 10. As has been mentioned, the scaling function of the DWT analysis is just a window function. It will give the similar statistical result as a Gaussian window if the orthogonality and completeness are not the key of the relevant statistics.

Figure 11 gives the one-point distribution $f\left(\Delta v_{i}\right)$ on scales $j=2-7$ of the SCDM. Models of $\tau \mathrm{CDM}$ and $\Lambda \mathrm{CDM}$ also give the similar results. The one-point distributions $f\left(\Delta v_{i}\right)$ are also scale-dependent, but it is significantly different from $f\left(v_{i}\right)$. On large scale $j=2,3$ the distribution $f\left(\Delta v_{i}\right)$ is approximately exponential, i.e. $\ln f\left(\Delta v_{i}\right)$ vs. $\Delta v_{i}$ can roughly be approximated by a straight line. However, on all scales $j \geq 5$, the distribution $f\left(\Delta v_{i}\right)$ is nothing but typically lognormal, i.e. $\ln \left[f\left(\Delta v_{i}\right) / f\left(2^{a} \Delta v_{i}\right)\right]=a \ln \left[f\left(\Delta v_{i}\right) / f\left(2 \Delta v_{i}\right)\right]$, where $a$ is a real number. Lognormal random field is often employed to model the non-linear clustering (Cole \& Jones, 1991). The mass field traced by the Ly $\alpha$ forests is also lognormal (Bi \& Davidsen, 1997; Feng \& Fang 2000). Moreover, lognormal random field is typically intermittent (e.g. Zel'dovich, Ruzmaikin, \& Sokoloff 1990). Based on these considerations, it is not unusual to find that the one-point distribution of $\Delta v_{i}$ is lognormal.

We also calculate the one-point distribution of pairwise velocity measured by conventional pair 
counting. The result is ploted in Fig. 12, which are the same as all measurements by pair counting method, i.e. the distributions basically are exponential. Although we have $\left\langle v_{12}^{2}(r)\right\rangle^{1 / 2} \simeq \sqrt{3} \sigma_{j}^{p v_{i}}$ on small scales, the PDF of $v_{12}(r)$ is very different from $\Delta v_{i}$ in the same scale range.

\section{Correlations between velocity and density fields}

\subsection{Local velocity-density correlation}

In redshift distortion theory, $\mathrm{VD}, \sigma^{v}$, and its distribution generally are assumed to be independent of the density field. Observational evidences, however, seem to support the existence of correlation between the velocity and local number density of galaxies. For instance, the pairwise velocity dispersion measured by the conventional method is found to be sensitive to the presence of dense objects, like rich clusters (e.g. Mo, Jing \& Börner 1993). However, the conventional method of measuring the peculiar or pairwise velocity is biased to dense objects, due to the number-counting and pair-counting-weighted statistic. Thus, the $v(\mathbf{x})-n(\mathbf{x})$ correlation might be contaminated by the density-density $n(\mathbf{x})-n(\mathbf{x})$ correlation. With the field variables $v_{i} \mathbf{j}, \mathbf{l}$ and $\Delta v_{i} \mathbf{j}, \mathbf{l}$, one can distinguish among the correlations of $v(\mathbf{x})-n(\mathbf{x})$ and $n(\mathbf{x})-n(\mathbf{x})$. Thus, one can detect the correlation between the velocity and local density without bias.

Let us consider the number-counting statistics of the mean peculiar velocity. It is given by

$$
v_{i \text { trad }}=\frac{\sum_{n=1}^{N_{g}} w_{n} v_{i}\left(\mathbf{x}_{\mathbf{n}}\right)}{\sum_{n=1}^{N_{g}} w_{n}}
$$

Using the "partition of unity" of wavelet (Daubechies 1992), i.e. $\sum_{\mathbf{l}=0}^{2^{\mathbf{j}}-1} \phi_{\mathbf{j}, \mathbf{l}}^{P}(\mathbf{x})=$ $\left(L_{1} L_{2} L_{3}\right) / 2^{j_{1}+j_{2}+j_{3}}$, one can rewrite eq. (52) as

$$
v_{\text {trad }}=\frac{\sum_{\mathbf{l}=0}^{2^{\mathbf{j}}-1} \sum_{n=1}^{N_{g}} w_{n} v\left(\mathbf{x}_{\mathbf{n}}\right) \phi_{\mathbf{j}, \mathbf{l}}\left(\mathbf{x}_{\mathbf{n}}\right)}{\sum_{\mathbf{l}=0}^{2 \mathbf{j}-1} \sum_{n=1}^{N_{g}} w_{n} \phi_{\mathbf{j}, \mathbf{l}}\left(\mathbf{x}_{\mathbf{n}}\right)}=\frac{\sum_{\mathbf{l}=0}^{2^{\mathbf{j}}-1} \epsilon_{\mathbf{j}, \mathbf{l}}^{v_{i}}}{\sum_{\mathbf{l}=0}^{2^{\mathbf{j}}-1} \epsilon_{\mathbf{j}, \mathbf{l}}^{n}}
$$

Therefore, the existence of the local $v-n$ correlation can be tested by

$$
C_{j \operatorname{trad}}^{\left(v^{2}, n^{2}\right)}=\frac{2^{\mathbf{j}} \sum_{\mathbf{l}=0}^{2^{\mathbf{j}}-1}\left[\epsilon_{\mathbf{j}, \mathbf{l}}^{v_{i}} \epsilon_{\mathbf{j}, \mathbf{l}}^{n}\right]^{2}}{\sum_{\mathbf{l}=0}^{2^{\mathbf{j}}-1}\left|\epsilon_{\mathbf{j}, 1}^{v_{i}}\right|^{2} \sum_{\mathbf{l}=0}^{2^{\mathbf{j}}-1}\left|\epsilon_{\mathbf{j}, 1}^{n}\right|^{2}}
$$


where we still consider the diagonal modes $j_{1}=j_{2}=j_{3}=j$. If there is no correlation between the local velocity variable $\epsilon_{\mathbf{j}, 1}^{v_{i}}$ and density variable $\epsilon_{\mathbf{j}, 1}^{n}$, we have $C_{j}^{\left(v^{2}, n^{2}\right)}=1$. We display the result in Fig. 13, which shows that for the CDM model $C_{j}^{\left(v^{2}, n^{2}\right)} \gg 1$ on $k>1 \mathrm{~h} \mathrm{Mpc}^{-1}$. Nevertheless, we should not immediately conclude the existence of strong correlation between $v(\mathbf{x})$ and $n(\mathbf{x})$, because Fig. 13 also shows a strong deviation from $C_{j}^{\left(v^{2}, n^{2}\right)}=1$ for random2, i.e. the samples without velocity-density correlation. That is, the $C_{j \text { trad }}^{\left(v^{2}, n^{2}\right)} \gg 1$ given by random2 is completely from density-density correlation.

To identify the $v-n$ correlation, it is essentially to refer to both random 1 and random 2 . The values of $C_{j}^{\left(v^{2}, n^{2}\right)}$ for the CDM mode deviate not only from random1, but also random2. Thus, we may conclude that the peculiar velocity given by the number-counting statistics is correlated with density field, but not very strong.

For the DWT velocity variables $v_{i} \mathbf{j}, \mathbf{l}$ [eqs.(19) and (36)], the mean peculiar velocity is $\left(1 / 2^{\mathbf{j}}\right) \sum_{\mathbf{l}=0}^{2^{\mathbf{j}}-1} \epsilon_{\mathbf{j}, 1}^{v_{i}} / \epsilon_{\mathbf{j}, \mathbf{l}}^{n}$. Thus, the local $v-n$ correlation can be measured by

$$
C_{j}^{\left(v^{2}, n^{2}\right)}=\frac{2^{\mathbf{j}} \sum_{\mathbf{l}=0}^{2^{\mathbf{j}}-1}\left[v_{i} \mathbf{j}, 1 \epsilon_{\mathbf{j}, 1}^{n}\right]^{2}}{\sum_{\mathbf{l}=0}^{2^{\mathbf{j}}-1}\left|v_{i} \mathbf{j}, \mathbf{l}\right|^{2} \sum_{\mathbf{l}=0}^{2 \mathbf{j}-1}\left|\epsilon_{\mathbf{j}, \mathbf{l}}^{n}\right|^{2}}=\left(\frac{\bar{\sigma}_{j}^{v}}{\sigma_{j}^{v}}\right)^{2} .
$$

That is, the ratio between $\bar{\sigma}_{j}^{v}$ and $\sigma_{j}^{v}$ is a measure of the VD - $n$ correlation. The CDM spectra of $\sigma_{j}^{v}$ and $\bar{\sigma}_{j}^{v}$ shown in Figs. 3 and 4 are very similar. Therefore, in term of the DWT variables, the VD- $n$ correlation is very weak (see Fig. 13).

The traditional estimation of the velocity dispersion within the patches $(\mathbf{j}, \mathbf{l})$ is given by

$$
\left|\Delta v_{i \text { trad }}\right|=\frac{\sum_{\mathbf{l}=0}^{2^{\mathbf{j}}-1}\left|\tilde{\epsilon}_{\mathbf{j}, \mathbf{l}}^{v_{i}}\right|}{\sum_{\mathbf{l}=0}^{2^{\mathbf{j}}-1} \epsilon_{\mathbf{j}, \mathbf{l}}^{n}} .
$$

It actually is the mean value of the absolute pairwise velocity of models $(\mathbf{j}, \mathbf{l})$. The local correlation between this pairwise velocity and density field can be estimated by

$$
C_{j \text { trad }}^{\left(\text {pv }^{2}, n^{2}\right)}=\frac{2^{\mathbf{j}} \sum_{\mathbf{l}=0}^{2^{\mathbf{j}}-1}\left[\tilde{\epsilon}_{\mathbf{j}, \mathbf{l}}^{v_{\mathbf{j}}, \mathbf{l}}\right]^{n}}{\sum_{\mathbf{l}=0}^{2^{\mathbf{j}}-1}\left|\tilde{\epsilon}_{\mathbf{j}, \mathbf{l}}^{v_{i}}\right|^{2} \sum_{\mathbf{l}=0}^{2^{\mathbf{j}}-1}\left|\epsilon_{\mathbf{j}, \mathbf{l}}^{n}\right|^{2}} .
$$

The result of $C_{j \text { trad }}^{\left(p v^{2}, n^{2}\right)}$ is given in Fig. 14. Although for the CDM model, the correlation $C_{j}^{\left(p v^{2}, n^{2}\right)}$ is much larger than 1 on small scales, but it is always less than the $C_{j \text { trad }}^{\left(p v^{2}, n^{2}\right)}$ of random2. Therefore, it seems to be not an evidence of the correlation between pairwise velocity and local density. 
Using the field-variable-defined pairwise velocity $\Delta v_{i} \mathbf{j}, \mathbf{l}$, the correlation should be can be measured by

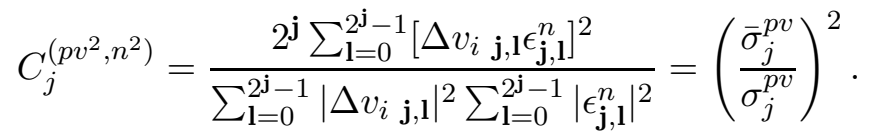

Fig 14 also displays this ratio. For the CDM models, $C_{j}^{\left(p v^{2}, n^{2}\right)}$ deviates from $C_{j}^{\left(p v^{2}, n^{2}\right)}=1$ (random1), but it is similar to that of random2. Therefore, no strong correlation between the pairwise velocity and local density can be identified.

\subsection{Correlation between PVD and local density fluctuations}

To account for the redshift distortion effect in measuring the DWT power spectrum, it would be necessary to investigate the correlation between the density fluctuations $\delta(\mathbf{x})$ and velocity field $\mathbf{v}(\mathbf{x})$. The DWT power spectrum is determined by the density fluctuations on mode $(\mathbf{j}, \mathbf{l})$. The bulk velocity of mode $(\mathbf{j}, \mathbf{l})$ causes only a position shift of mode $(\mathbf{j}, \mathbf{l})$ in redshift space with respect to real space, but do not change the corresponding power of the density fluctuations of mode $(\mathbf{j}, \mathbf{l})$. On the other hand, the pairwise velocity of mode $(\mathbf{j}, \mathbf{l})$ will change the power of the density fluctuations of mode $(\mathbf{j}, \mathbf{l})$. Therefore, the redshift distortion of the DWT power spectrum is mainly caused by velocity field variables $\Delta \mathbf{v}_{\mathbf{j}, \mathbf{l}}$, not $\mathbf{v}_{\mathbf{j}, \mathbf{l}}$. We will only study the correlation between $\Delta \mathbf{v}_{\mathbf{j}, \mathbf{l}}$ and density fluctuation $\delta(\mathbf{x})$.

A simplest statistical measure of the $\Delta \mathbf{v}_{\mathbf{j}, 1^{-}} \delta$ correlation is the second order statistic defined as

$$
C_{j}^{(p v, \delta)}=\frac{2^{\mathbf{j}} \sum_{\mathbf{l}=0}^{2^{\mathbf{j}}-1} \tilde{\epsilon}_{\mathbf{j}, 1}^{v^{i}} \tilde{\epsilon}_{\mathbf{j}, \mathbf{l}}^{n}}{\sum_{\mathbf{l}=0}^{2^{\mathbf{j}}-1}\left|\tilde{\epsilon}_{\mathbf{j}, \mathbf{1}}^{v^{i}}\right| \sum_{\mathbf{l}=0}^{2^{\mathbf{j}}-1}\left|\tilde{\epsilon}_{\mathbf{j}, \mathbf{1}}^{n}\right|},
$$

$C^{(p v, \delta)}$ will be equal to zero, if there is no correlation between velocity fluctuation WFC $\tilde{\epsilon}_{\mathbf{j}, 1}^{v_{i}}$ and density fluctuation WFC $\tilde{\epsilon}_{\mathbf{j}, 1}^{n}$.

Fig. 15 shows $C_{j}^{(p v, \delta)}$ in a CDM model, all results are consistent with $C_{j}^{(p v, \delta)}=0$ within the error bars. Thereby, in terms of second order statistics, it is likely reasonable assumption that there is no $\Delta \mathbf{v}_{\mathbf{j}, 1^{-}} \delta$ correlation. However, $C_{j}^{(p v, \delta)}=0$ doesn't mean that $\Delta \mathbf{v}_{\mathbf{j}, 1}$ and $\delta(\mathbf{x})$ are uncorrelated in general. Since the one-point distribution of $\Delta \mathbf{v}_{\mathbf{j}, 1}$ are invariant with respect to 
$\Delta v_{i}(\mathbf{x}) \rightleftharpoons-\Delta v_{i}(\mathbf{x})$, in this case, statistics with linear term of $\Delta v_{i}(\mathbf{x})$, like $\left\langle\Delta v_{i}(\mathbf{x}) \delta(\mathbf{x})\right\rangle$ will be zero. Therefore, we should study the correlation between $\left|\Delta v_{i}(\mathbf{x})\right|^{2}$ and $\delta^{2}(\mathbf{x})$, which can be calculated by

$$
C_{j}^{\left(p v^{2}, \delta^{2}\right)}=\frac{2^{\mathbf{j}} \sum_{\mathbf{l}=0}^{2^{\mathbf{j}}-1}\left[\tilde{\epsilon}_{\mathbf{j}, \mathbf{l}}^{v^{i}} \tilde{\epsilon}_{\mathbf{j}, \mathbf{l}}^{n}\right]^{2}}{\sum_{\mathbf{l}=0}^{2^{\mathbf{j}}-1}\left|\tilde{\epsilon}_{\mathbf{j}, \mathbf{l}}^{v^{i}}\right|^{2} \sum_{\mathbf{l}=0}^{2^{\mathbf{j}}-1}\left|\tilde{\epsilon}_{\mathbf{j}, \mathbf{l}}^{n}\right|^{2}} .
$$

The results for the dark matter model are also plotted in Fig. 15. It shows that $C_{j}^{\left(p v^{2}, \delta^{2}\right)}$ of the CDM models is higher than that of random1 and random2. Therefore, $\Delta \mathbf{v}$ and $\delta(\mathbf{x})$ are locally correlated on scales $k>0.2 \mathrm{~h} \mathrm{Mpc}^{-1}$.

\section{Conclusion}

It is known that in the DWT representation a continuous field, like cosmic density field $n(\mathbf{x})$, can be described by discrete variables, given by the DWT scale-space decomposition of $n(\mathbf{x})$ into the DWT mode $(\mathbf{j}, \mathbf{l})$ (Fang \& Feng 2000). Since the modes $(\mathbf{j}, \mathbf{l})$ are complete and orthogonal, the DWT decomposition is information-lossless and not redundant. It insures that one can compare statistical results of different samples.

Taking the advantage of the DWT analysis, we show that the cosmic velocity field can also be properly described by the discrete variables $v_{i} \mathbf{j}, \mathbf{l}$, which are given by an assignment of the number density and velocity of galaxies (or particles) into the DWT modes $(\mathbf{j}, \mathbf{l})$. In this scheme, the peculiar velocity and pairwise velocity of galaxies or particles are given by field variables, which correspond to the coefficients of the scaling functions and wavelets of the DWT decomposition. As a consequence, the VD and PVD are no longer measured by number-counting or pair-counting statistic, but with the ensemble of the field variables.

Using simulation samples, we show that peculiar velocity field is significantly different from randomized field. The peculiar velocities show correlation on scales of few tens $\mathrm{h}^{-1} \mathrm{Mpc}$. The pairwise velocity (or relative velocity) has similar correlation. On small scales, we also found significant correlations between pairwise velocity and density fluctuations. This is especially valuable in treating the redshift distortion of the DWT power spectrum. 
Although the VD and PVD look similar to each other in some aspects, they actually are very different both statistically and physically. The VD-to-PVD ratio shows the difference between the scale-dependencies of the VD and PVD. More prominent difference between the VD and PVD is shown by one-point distribution. The one-point distribution of the VD is approximately exponential, while the PVD is lognormal. This difference is typical of intermittent field (a similar difference between the one-point distributions of field variable $A$ and the difference $\Delta A$ in turbulence is given by Shraiman \& Sigglia 2000). Therefore, one can conclude that the cosmic velocity field is intermittent.

LLF and YQC acknowledges support from the National Science Foundation of China (NSFC) and National Key Basic Research Science Foundation.

\section{A. Poisson noise}

The observed galaxy number density distribution $n(\mathbf{x})$ [eq.(2)] is believed to be a realization of a Poisson point process with an intensity $\tilde{n}(\mathbf{x})=\bar{n}^{g}(\mathbf{x})[1+\delta(\mathbf{x})]$, where $\bar{n}^{g}(\mathbf{x})$ is selection function, and $\delta(\mathbf{x})=\left[n(\mathbf{x}) / \bar{n}^{g}(\mathbf{x})\right]-1$ is the density contrast fluctuations of the underlying matter field. Thus, the correlation function of $\delta(\mathbf{x})$ is

$$
\langle\delta(\mathbf{x}) \delta(\mathbf{x})\rangle=-1+\left\langle\frac{n(\mathbf{x}) n\left(\mathbf{x}^{\prime}\right)}{\bar{n}^{g}(\mathbf{x}) \bar{n}^{g}\left(\mathbf{x}^{\prime}\right)}\right\rangle-\delta_{D}\left(\mathbf{x}-\mathbf{x}^{\prime}\right) \frac{1}{\bar{n}^{g}\left(\mathbf{x}^{\prime}\right)} .
$$

The power spectrum of $\delta(\mathbf{x})$ is then given by (Fang \& Feng 2000, Yang et al. 2001)

$$
P_{\mathbf{j}}=I_{\mathbf{j}}^{2}-N_{\mathbf{j}}
$$

where

$$
I_{\mathbf{j}}^{2}=\frac{1}{2^{\left(j_{1}+j_{2}+j_{3}\right)}} \sum_{l_{1}=0}^{2^{j_{1}}-1} \sum_{l_{2}=0}^{2^{j_{2}}-1} \sum_{l_{3}=0}^{2^{j_{3}}-1} \frac{\left[\tilde{\epsilon}_{\mathbf{j}, 1}^{n}\right]^{2}}{\left[\bar{n}_{\mathbf{j}, 1}^{g}\right]^{2}}
$$

and

$$
N_{\mathbf{j}}=\frac{1}{2^{\left(j_{1}+j_{2}+j_{3}\right)}} \sum_{l_{1}=0}^{2^{j_{1}}-1} \sum_{l_{2}=0}^{2^{j_{2}}-1} \sum_{l_{3}=0}^{2^{j_{3}}-1} \frac{1}{\bar{n}_{\mathbf{j}, \mathbf{l}}^{g}} .
$$


where the WFC $\tilde{\epsilon}_{\mathbf{j}, 1}^{g}$ is given by

$$
\tilde{\epsilon}_{\mathbf{j}, 1}^{n}=\int n(\mathbf{x}) \psi_{\mathbf{j}, 1}(\mathbf{x}) d \mathbf{x}
$$

and $\bar{n}_{\mathbf{j}, 1}^{g}$ is the mean number density of the selection function $\bar{n}^{g}(\mathbf{x})$ in the mode $(\mathbf{j}, \mathbf{l})$.

The term $I_{\mathbf{j}}^{2}$ is the mean power of $\mathbf{j}$ modes measured from the observed realization $n^{g}(\mathbf{x})$, and the term $N_{\mathbf{j}}$ is the power on $\mathbf{j}$ modes due to the Poisson noise. Therefore, the Poisson error for the galaxy distribution $n(\mathbf{x})$ can be described by an error distribution as

$$
\begin{aligned}
& n^{\text {error }}(\mathbf{x})=\sum_{l_{1}=0}^{2^{J_{1}}-1} \sum_{l_{2}=0}^{J_{2}-1} \sum_{l_{3}=0}^{J_{3}-1} \\
& \quad\left[\frac{L_{1} L_{2} L_{3}}{2^{J_{1}+J_{2}+J_{3}}} \bar{n}_{\mathbf{j}, 1}^{g}\right]^{1 / 2} \delta^{D}\left(x_{1}-l_{1} L_{1} / 2^{J_{1}}\right) \delta^{D}\left(x_{2}-l_{2} L_{2} / 2^{J_{2}}\right) \delta^{D}\left(x_{3}-l_{3} L_{3} / 2^{J_{3}}\right) .
\end{aligned}
$$

where $\left(J_{1}, J_{2}, J_{3}\right)$ correspond to the smallest scale of sample considered. In the case of $\bar{n}^{g}(\mathbf{x})=$ const and $L_{1}=L_{2}=L_{3}=L, J_{1}=J_{2}=J_{3}=J$, Eq.(A6) becomes

$$
n^{\text {error }}(\mathbf{x})=\sum_{\mathbf{l}=0}^{2^{\mathbf{J}}-1} \sqrt{\frac{N_{g}}{2^{3 J}}} \delta^{D}\left(\mathbf{x}-\mathbf{l} L / 2^{J}\right)
$$

It simply means that the Poisson noise of the galaxy number in a cell $(\mathbf{J}, \mathbf{l})$ is $\sqrt{N_{g} / 2^{3 J}}$.

\section{B. Off-counting algorithm}

The typical terms in eqs.(41) and (43) are $\epsilon_{\mathbf{j}, 1}^{v_{i}} / \epsilon_{\mathbf{j}, 1}^{n}$ and $\tilde{\epsilon}_{\mathbf{j}, 1}^{v_{i}} / \epsilon_{\mathbf{j}, 1}^{n}$, where the denominator is given by eq.(39)

$$
\epsilon_{\mathbf{j}, 1}^{n}=\int n(\mathbf{x}) \phi_{\mathbf{j}, \mathbf{l}}(\mathbf{x}) d \mathbf{x}=\sum_{n=1}^{N_{g}} w_{n} \phi_{\mathbf{j}, 1}\left(\mathbf{x}_{n}\right) .
$$

Therefore, for modes $\mathbf{j}, \mathbf{l}$ corresponding to position of the voids of galaxies, i.e. $n(\mathbf{x})$ is very small or zero, the SFC $\epsilon_{\mathbf{j}, 1}^{n}$ would be very small, even zero. These modes will lead to very large term of $\epsilon_{\mathbf{j}, 1}^{v_{i}} / \epsilon_{\mathbf{j}, 1}^{n}$. Actually, these terms have very large error, because the number density $n(\mathbf{x})$ in these modes generally is less than the error distribution at the same spatial range $n^{\text {error }}(\mathbf{x})$.

To avoids the contamination of these large error term, we use the following algorithm.

1. Perform DWT for the number density distributions of $n(\mathbf{x})$, and $n^{\text {error }}$. 
2. The SFC of $n(\mathbf{x})$ generally is larger than the SFCs of $n^{\text {error }}(\mathbf{x})$, and therefore $\epsilon_{\mathbf{j}, 1}^{n}>\epsilon_{j l}^{\text {error }}$. However, for some modes $(\mathbf{j}, \mathbf{l})$, we have

$$
\epsilon_{j, l}^{n} \leq \epsilon_{\mathbf{j}, 1}^{e r r o r}
$$

These $(\mathbf{j}, \mathbf{l})$ are noise dominated modes, and no observed data are available.

3. We eliminate all modes affected by the noise dominated modes. This is, if $(\mathbf{j}, \mathbf{l})$ satisfies eq.(B2), we off-count the following modes

$$
\begin{aligned}
& j_{i}^{\prime} \geq j_{i} \\
& l_{i}^{\prime}=l 2^{j_{i}^{\prime}-j_{i}} \ldots(l+1) 2^{j_{i}^{\prime}-j_{i}}-1 .
\end{aligned}
$$

This is called off-counting algorithm. It is effectively to eliminate the effects of $\epsilon_{\mathbf{j}, 1}^{n} \simeq 0$, and give reasonable estimation of VD [eq.(41)] and PVD [eq.43)] on the same scale range as the power spectrum. 


\section{REFERENCES}

Bardeen, J.M., Bond, J.R., Kaiser, N. \& Szalay, A.S., 1986, ApJ, 304, 15

Bi, H.G. \& Davidson, 1997, ApJ, 479, 523

Coles, P \& Jones, B. 1991, MNRAS, 248, 1

Couchman, H.M.P., 1991, ApJ, 368, 23

Daubechies I. 1992, Ten Lectures on Wavelets, (Philadelphia, SIAM)

Davis, M. \& Peebles, P.J.E. 1983, ApJ, 267, 465

Diaferio, A. \& Geller, M.J. 1996, ApJ, 467, 19

Fang, L.Z. \& Thews, R. 1998, Wavelet in Physics, (World Scientific, Singapore)

Fang, L.Z. \& Feng, L.L. 2000, ApJ, 539, 5

Farge, M. 1992, Ann. Rev. Fluid. Mech. 24, 395

Feng, L.L. \& Fang, L.Z. 2000, ApJ, 535, 519

Feng, L.L. Pando, J. \& Fang, L.Z. 2001, ApJ, in press

Fisher, K.B., Davis, M., Strauss, M., Yahil, A. \& Huchra, J.P. 1994, MNRAS, 267, 927

Jamkhedkar, P., Zhan, H. \& Fang, L.Z. 2000, ApJ, 543, L1

Jing Y.P., Mo, H.J., \& Börner G., 1998, ApJ, 4941

Kaiser, N. 1987, MNRAS, 227, 1

Landy, S.D., Szalay, A.S., \& Broadhurst, T.J. 1998, ApJ, 494, L133.

Mallat, S.G. 1989a, IEEE Trans, on PAMI, 11, 674

Mallat, S.G. 1989b, Trans. Am. Math. Soc. 315, 69 
Meyer, Y. 1992, Wavelets and Operators, (Cambridge Press, New York).

Mo, H.J., Jing, Y.P. \& Börner, 1993, MNRAS, 264, 825

Pando, J. \& Fang, L.Z. 1998, Phys. Rev. E57, 3593

Pando, J. Feng, L.L. \& Fang, L.Z. 2001, ApJ, in press

Peacock, J.A. \& Dodds, S.J., 1994, MNRAS, 167, 1020

Rauzy, S., Lachieze-Rey, M. \& Henriksen, 1993, A\&A, 273, 357

Sheth, R.K. 1996, MNRAS, 279, 1310

Shraiman, B.I. \& Siggia, E.D. 2000, Nature, 405, 639

Strauss, M.A., Ostriker, J.P. \& Cen, R. 1998, ApJ, 494, 20

Suto, Y. \& Fujita, M. 1990, ApJ, 360, 7

Xu, W., Fang, L.Z. \& Wu, X.P. 2000, ApJ, 532, 728

Yang, X.H., Feng, L.L., Chu, Y.Q. \& Fang, L.Z. 2001, ApJ, 553, 1

Zel'dovich, Ya.B., Ruzmaikin, A.A. \& Sokoloff, D.D. 1990, The Almighty Chance, (World Scientific, Singapore)

Zhan, H. Jamkhedkar, P. \& Fang, L.Z. 2001, ApJ, in press 

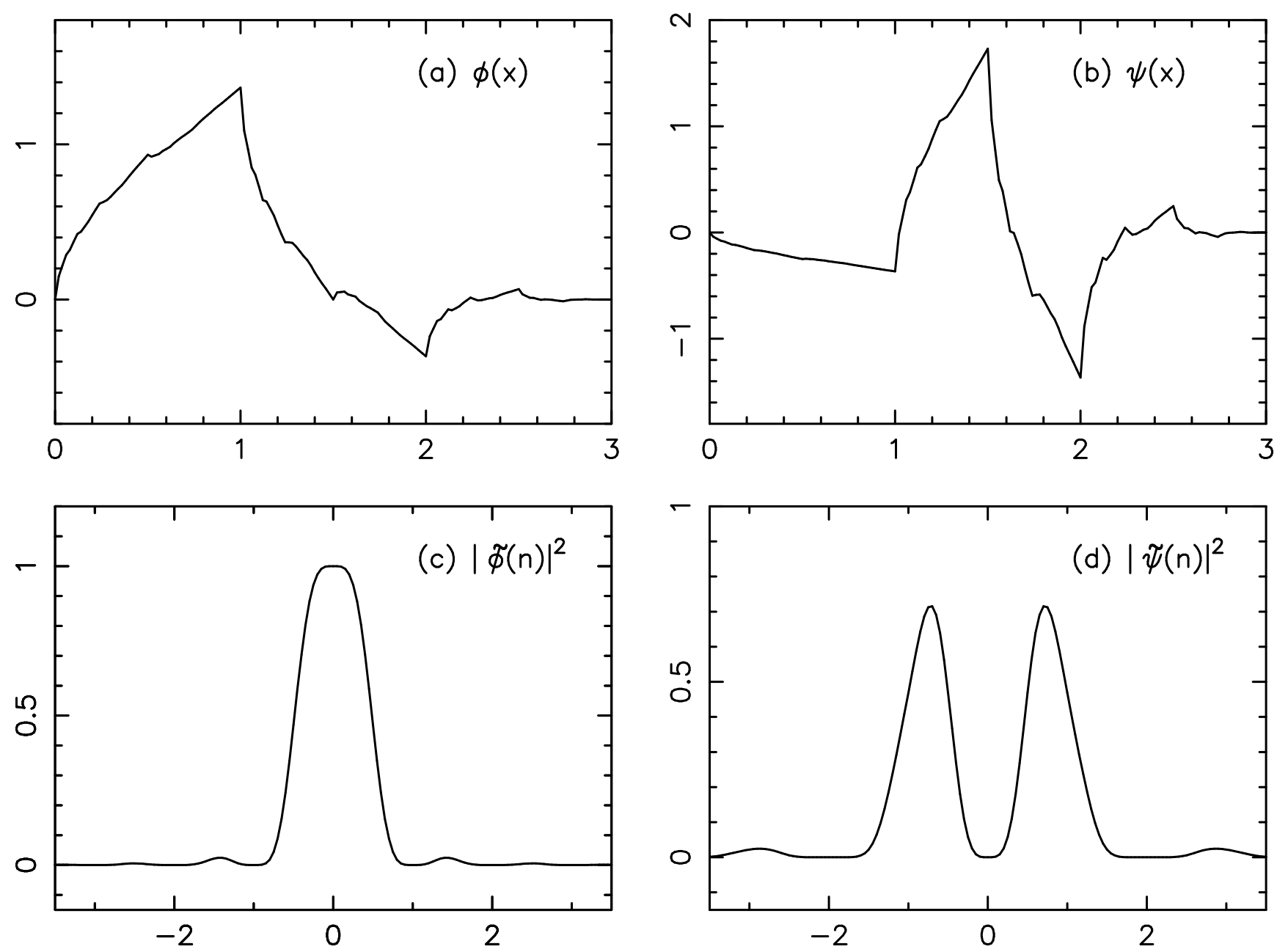

Fig. 1.- The basic scaling function (a), basic wavelet (b) and their Fourier transforms (c) and (d) of Daubechies 4 wavelet. 


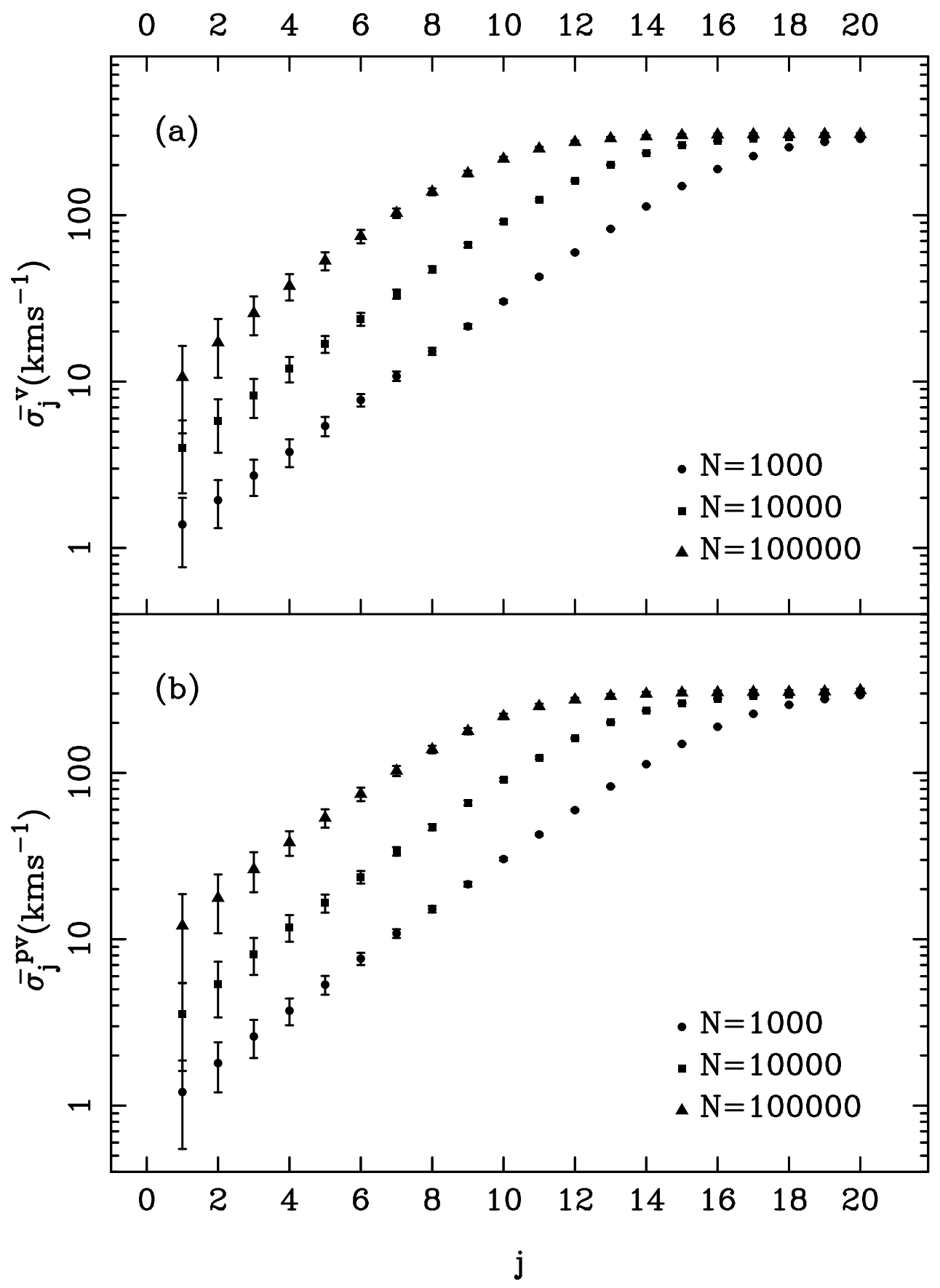

Fig. 2.- The VD spectrum, $\bar{\sigma}_{j}^{v}\left[\right.$ panel (a)] and the PVD spectrum, $\bar{\sigma}_{j}^{p v}[$ panel (b)] of 1-D random samples, which are uniform random distribution of $N$ particles in spatial range $L=1$. Each particles assigned with a velocity $v_{n}$ drawn from a Gaussian distribution with variance $300 \mathrm{~km} \mathrm{~s}^{-1}$. 


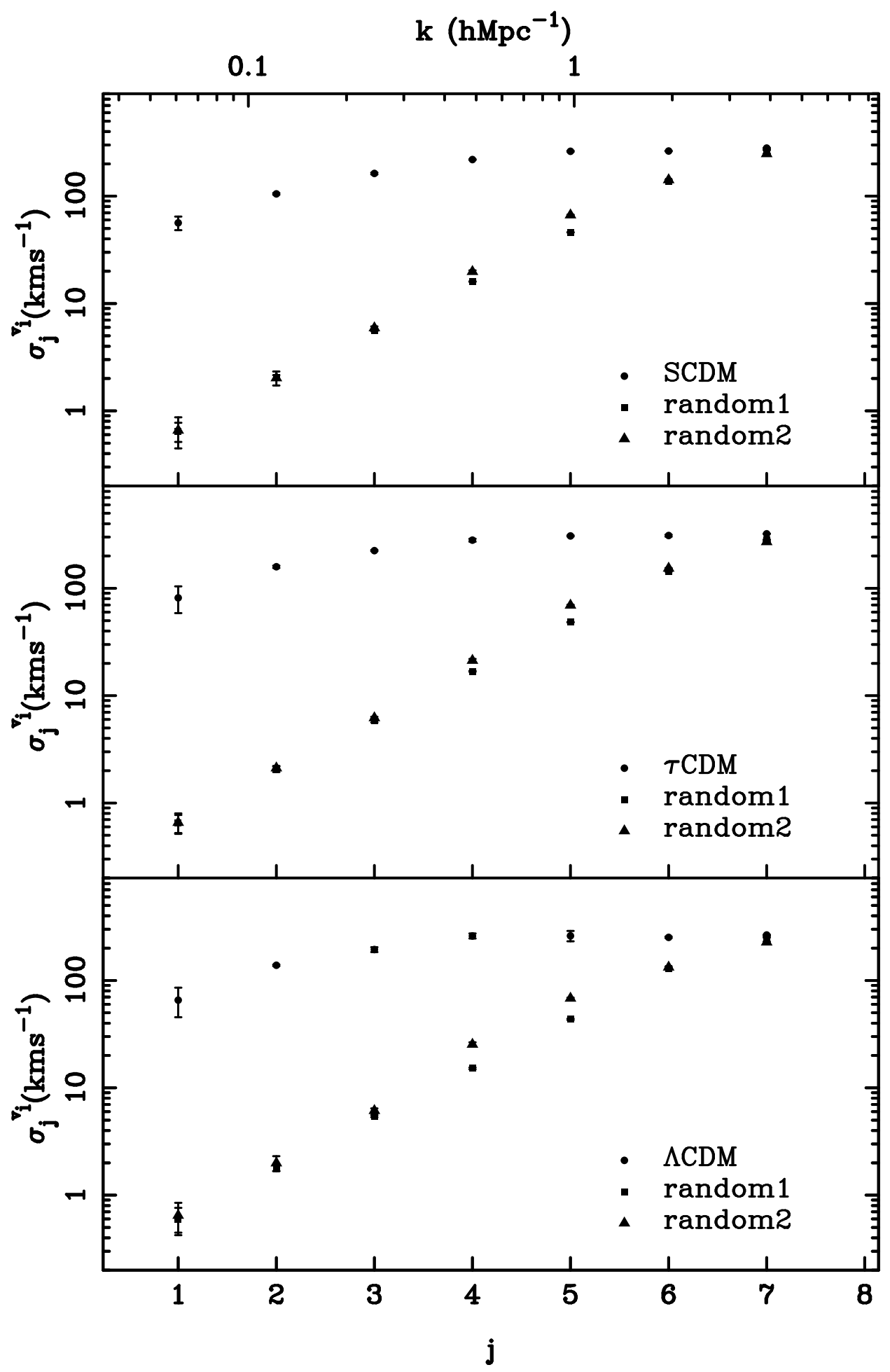

Fig. 3.- The VD spectrum of the diagonal mode, $\sigma_{j}^{v_{i}}$ of simulation samples for models SCDM (upper panel), $\tau \mathrm{CDM}$ (central panel) and $\Lambda \mathrm{CDM}$ (lower panel). The random1 samples are given by assigning $N$ velocities to randomly distributed particles, and random2 is produced by randomizing 


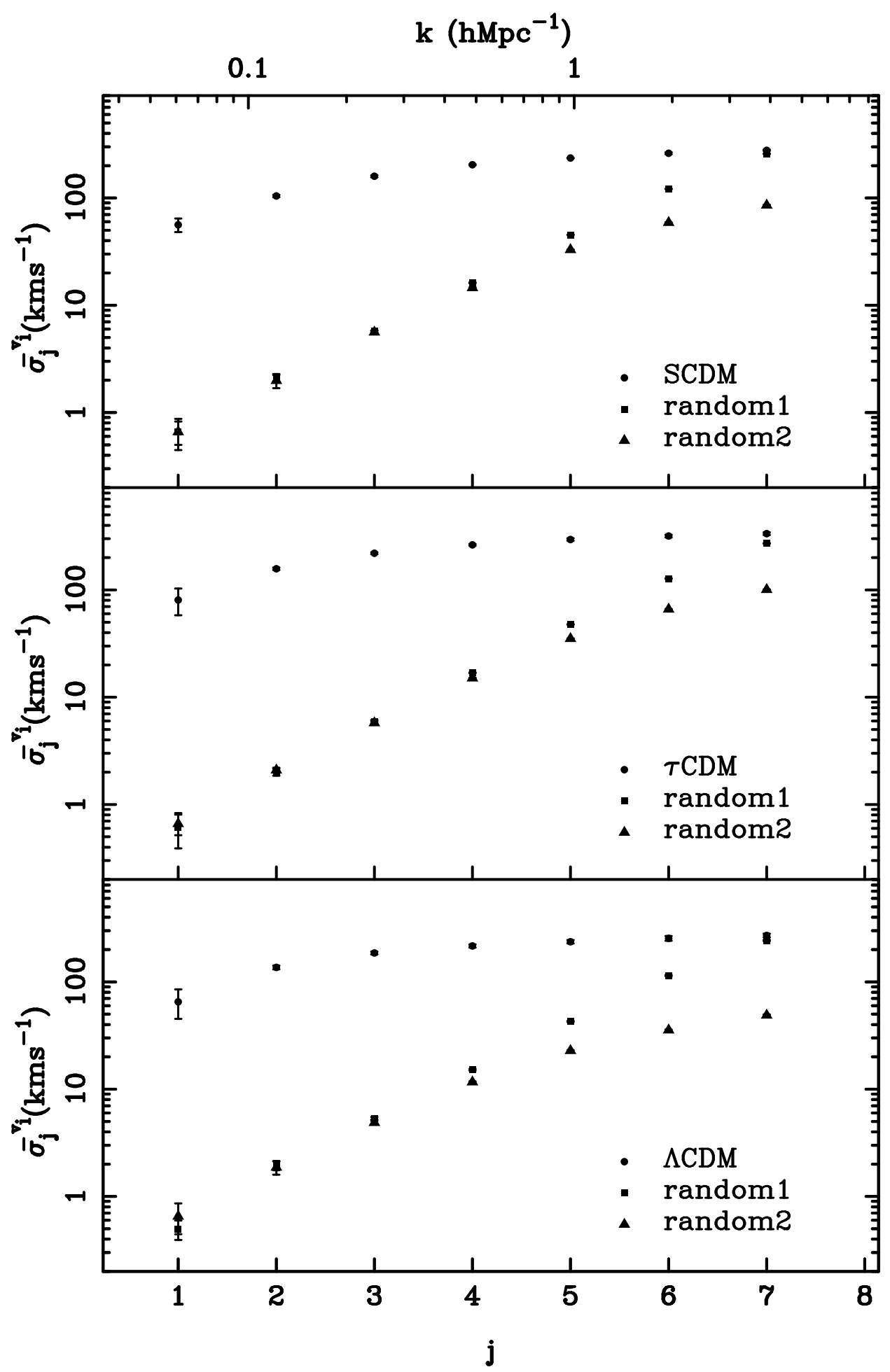

Fig. 4. - The modified VD spectrum, $\bar{\sigma}_{j}^{v_{i}}$ of diagonal mode $\left(j_{1}=j_{2}=j_{3}=j\right)$ for SCDM simulation samples (upper panel), $\tau$ CDM (central panel) and $\Lambda$ CDM (lower panel). The random samples are given by the randomizations as Fig.3. The error bars are 1- $\sigma$ variance from 10 realizations for each 


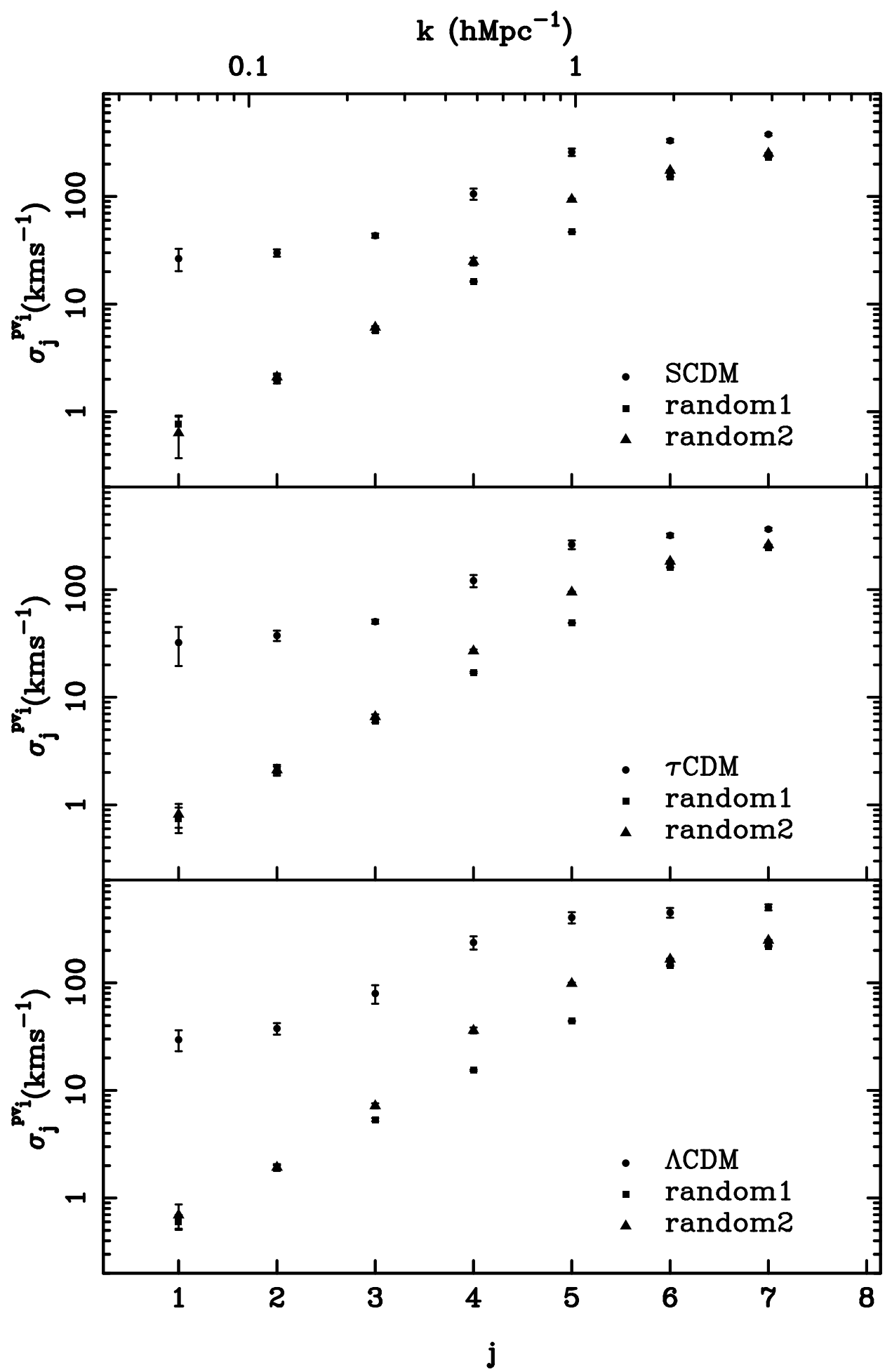

Fig. 5.- The PVD spectrum, $\sigma_{j}^{p v_{i}}$, of diagonal mode $\left(j_{1}=j_{2}=j_{3}=j\right)$ for SCDM simulation samples (upper panel), $\tau$ CDM (central panel) and $\Lambda$ CDM (lower panel). The random samples are given by the randomizations as Fig. 3. The error bars are 1- $\sigma$ variance from 10 realizations for 


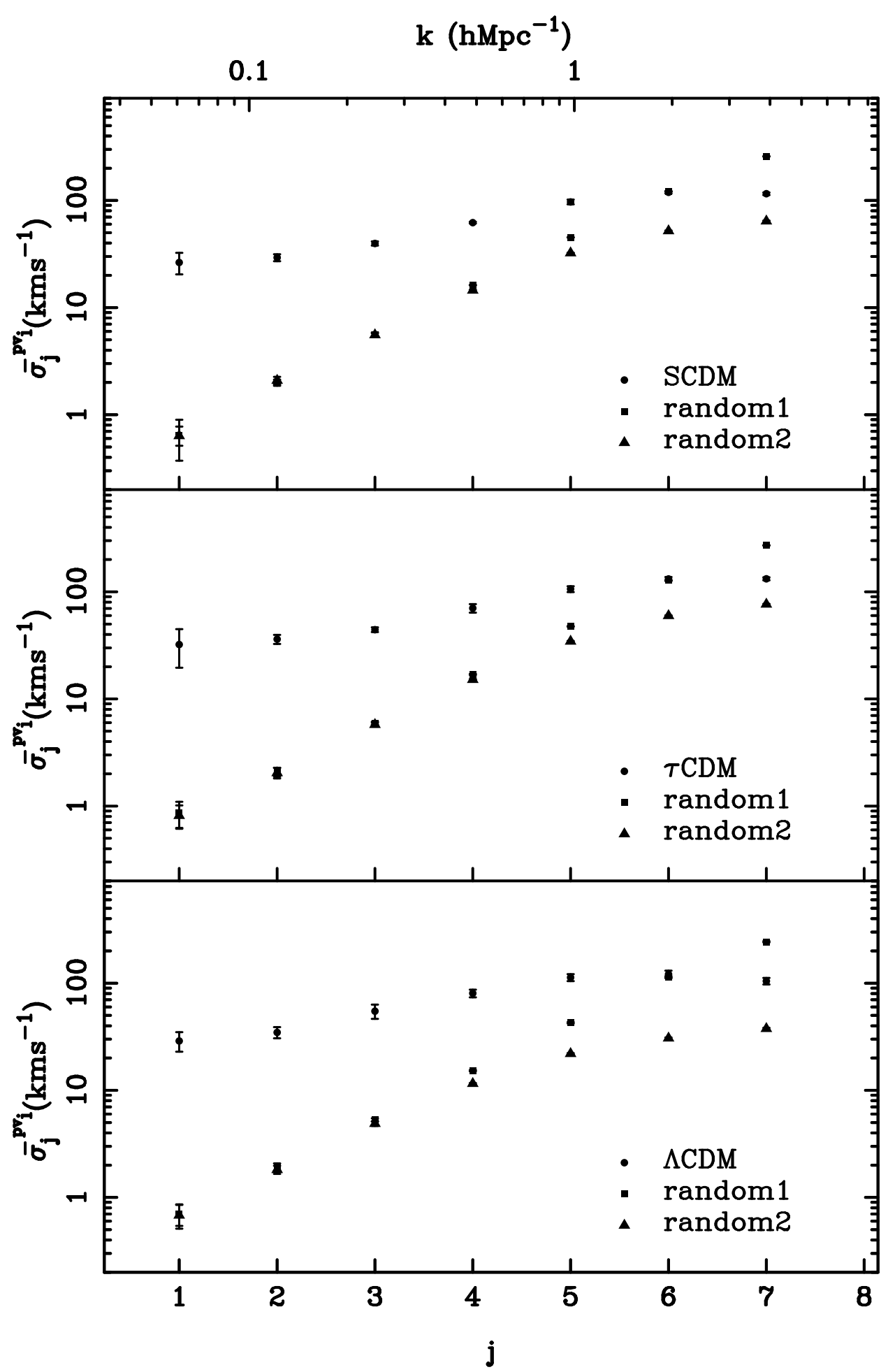

Fig. 6. - The modified PVD spectrum, $\bar{\sigma}_{j}^{p v_{i}}$, of diagonal mode $\left(j_{1}=j_{2}=j_{3}=j\right)$ for SCDM simulation samples (upper panel), $\tau \mathrm{CDM}$ (central panel) and $\Lambda \mathrm{CDM}$ (lower panel). The random samples are given by the randomizations as Fig. 3. The error bars are 1- $\sigma$ variance from 10 realizations for each model. 


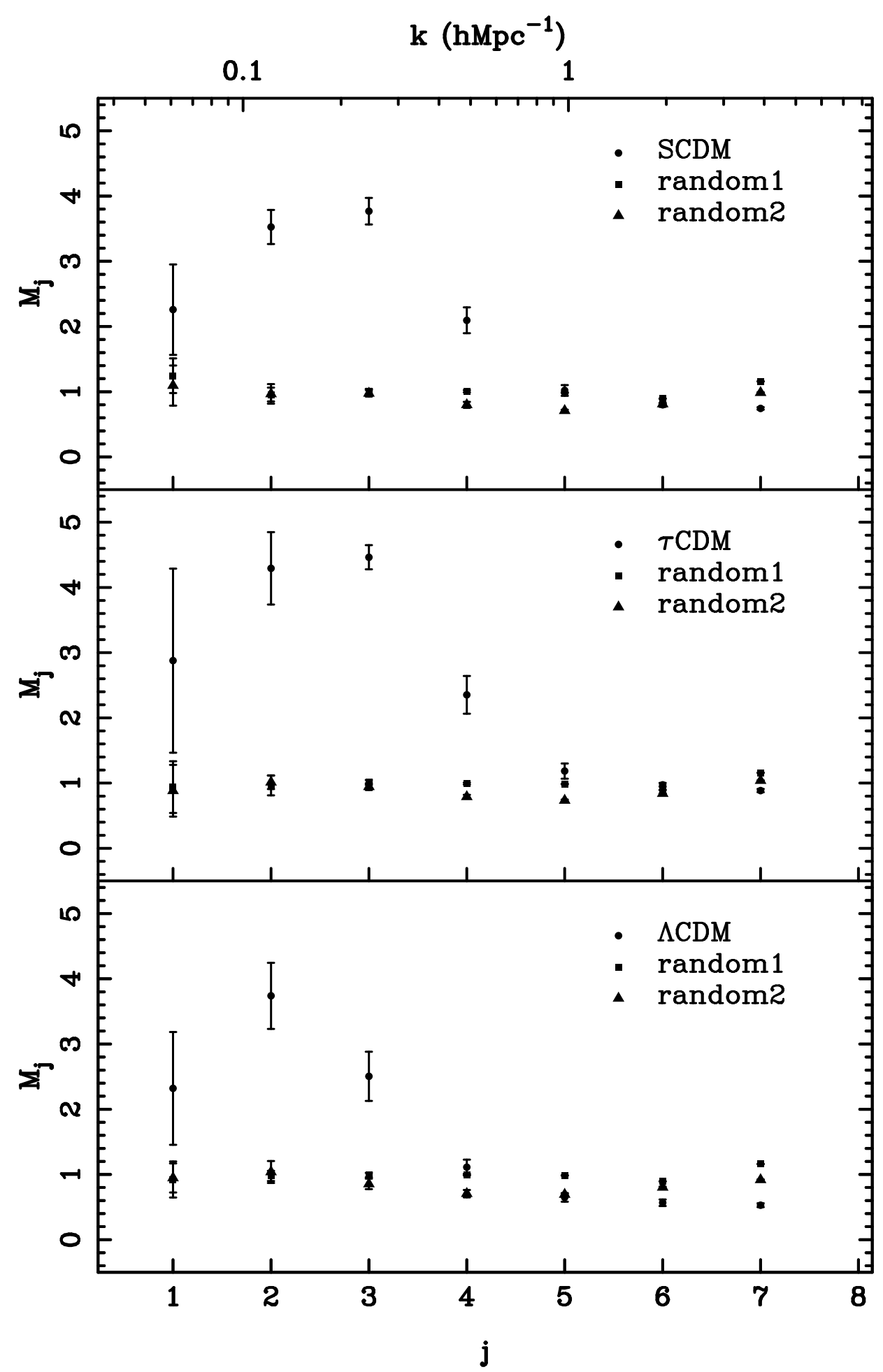

Fig. 7.- The VD-to-PVD ratio or Mach number $M_{j}$, of simulation samples for models SCDM (upper panel), $\tau \mathrm{CDM}$ (central panel) and $\Lambda \mathrm{CDM}$ (lower panel). The random samples are the same as Fig. 3. The error bars are 1- $\sigma$ variance from 10 realizations for each model. 


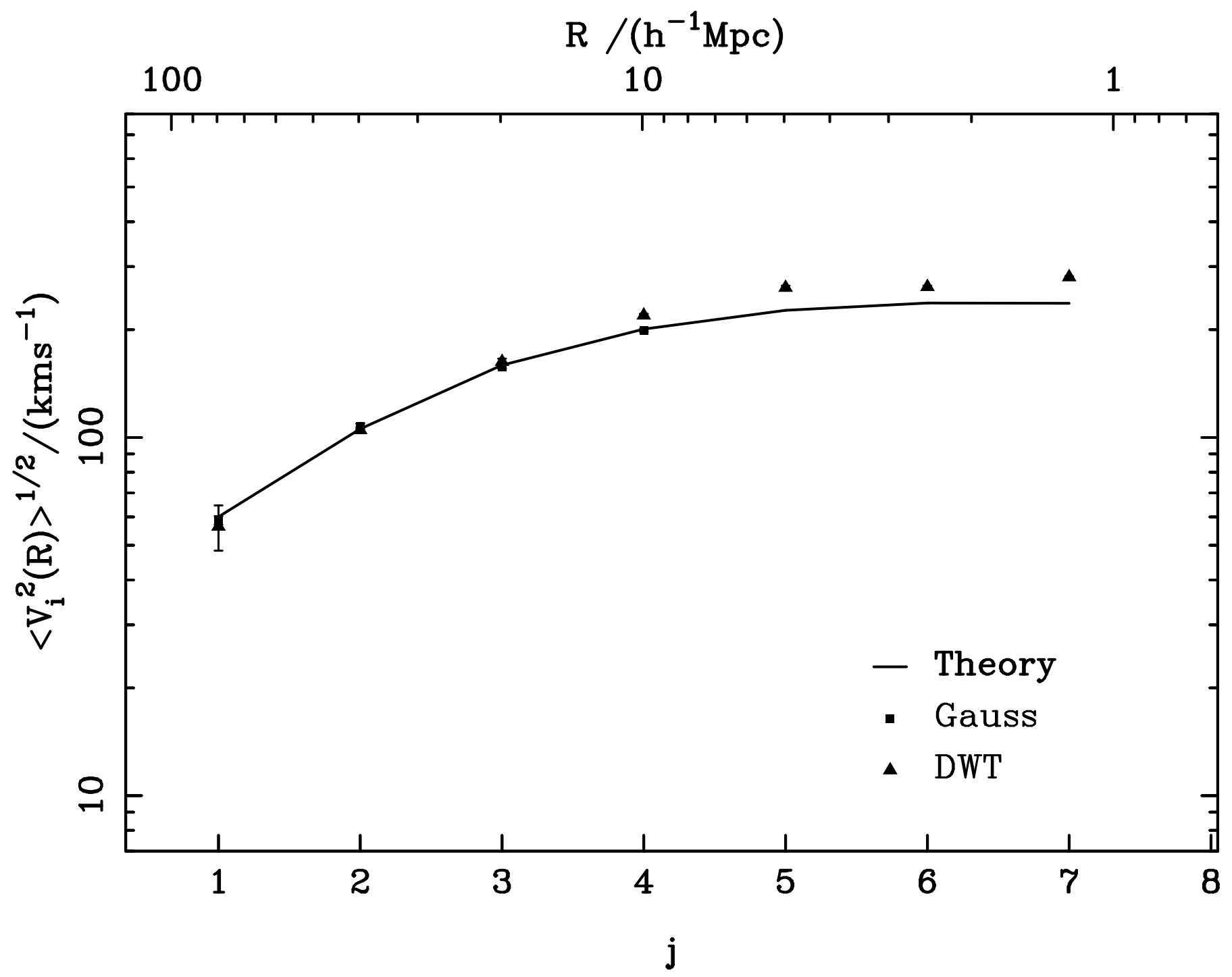

Fig. 8.- A comparison between the velocity dispersion $\left\langle V_{i}^{2}(R)\right\rangle^{1 / 2}$ measured by a Gaussian window function (square) and by the DWT method (triangle). The theory value (line) is the expected linear regime velocity using eq. (49). $R$ is the size of the window. 


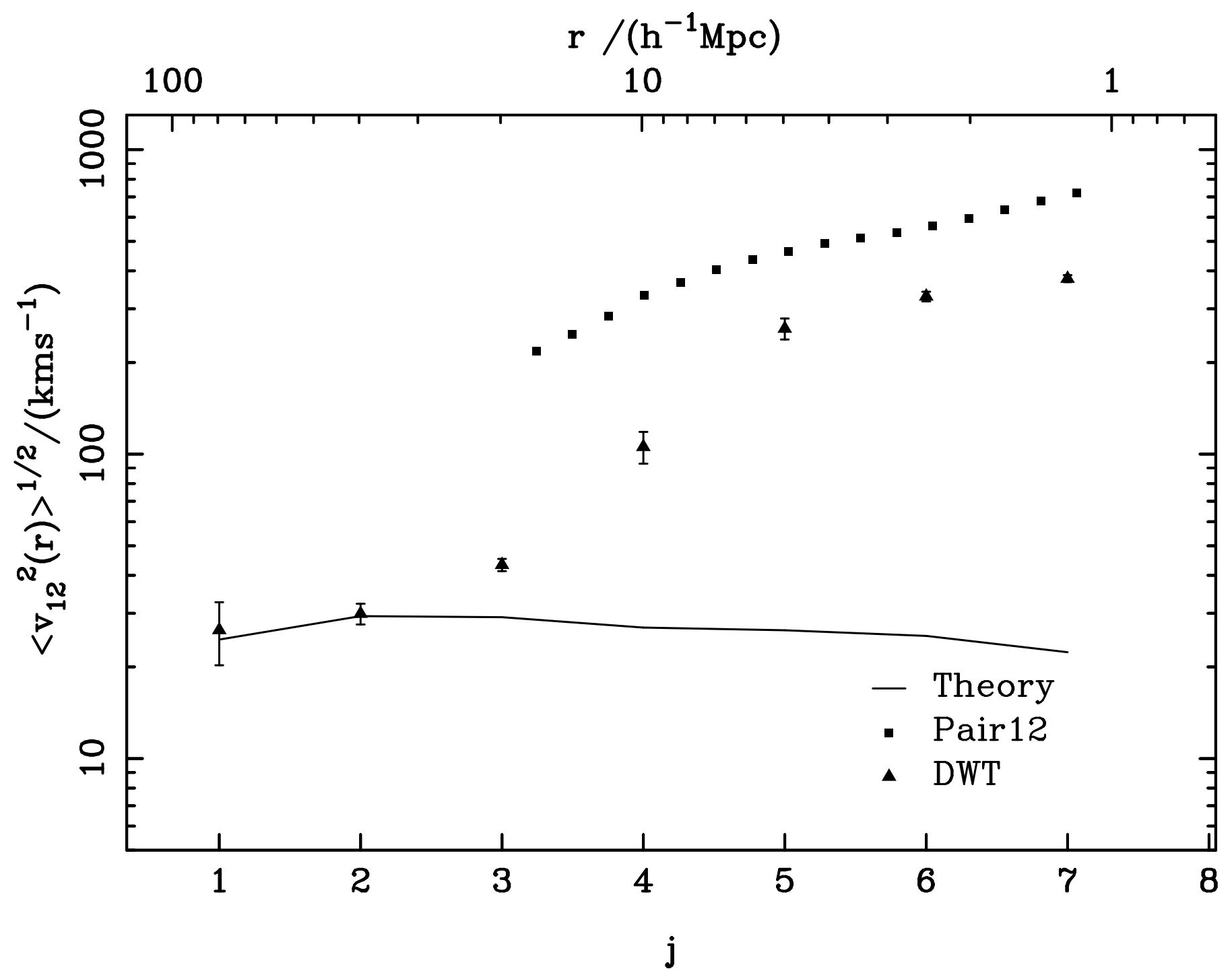

Fig. 9. - A comparison between the pair velocity dispersion $\left\langle v_{12}^{2}(r)\right\rangle^{1 / 2}$ measured by a traditional pair counting method (square) and by the DWT method (triangle). $r$ is the distance of the pairs. The theory value (line) is calculated by eq. (51). 

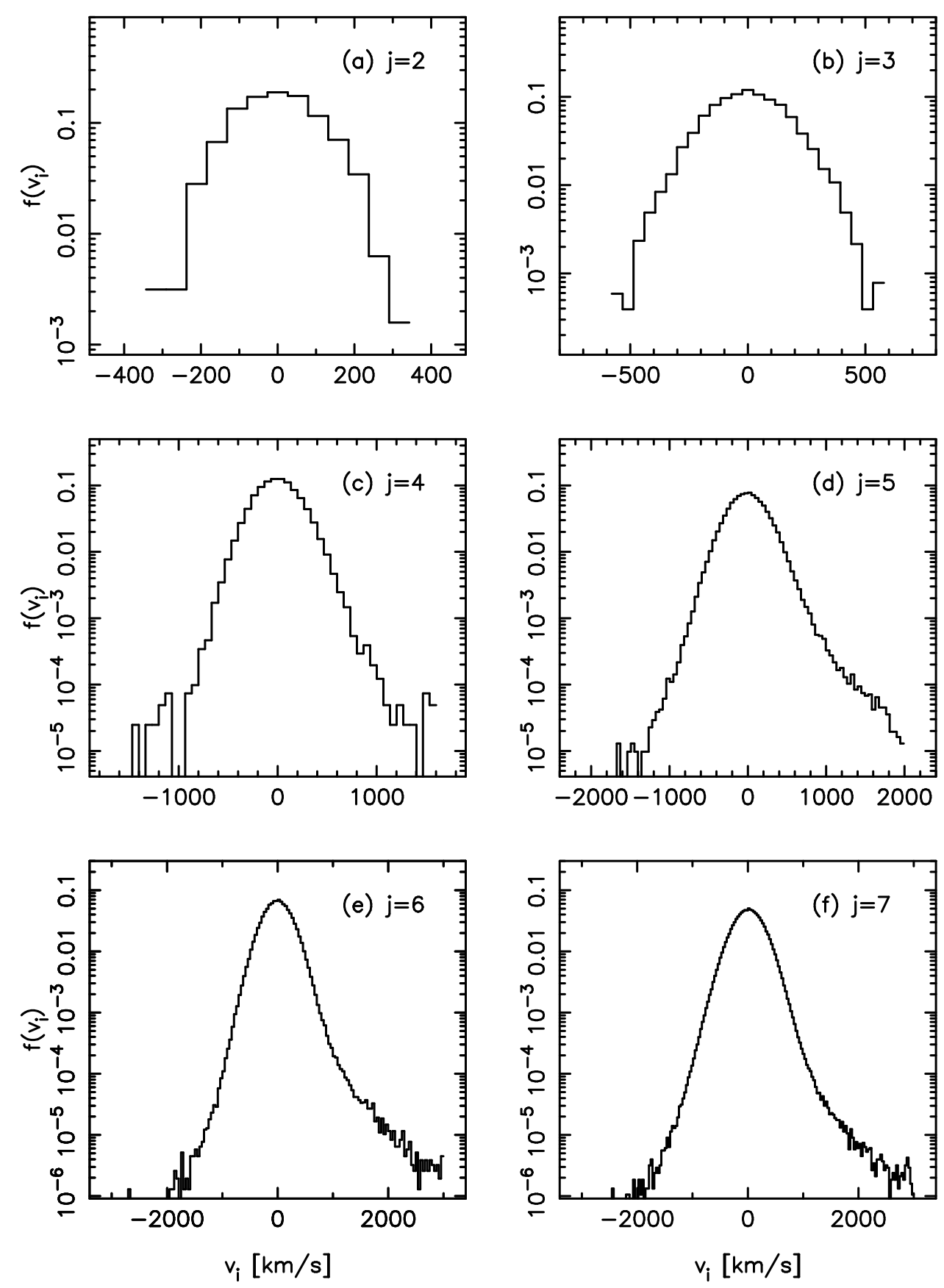

Fig. 10.- The one-point distribution of $v_{i}$ on scales $j=2-7$ for simulation samples of the SCDM model. 

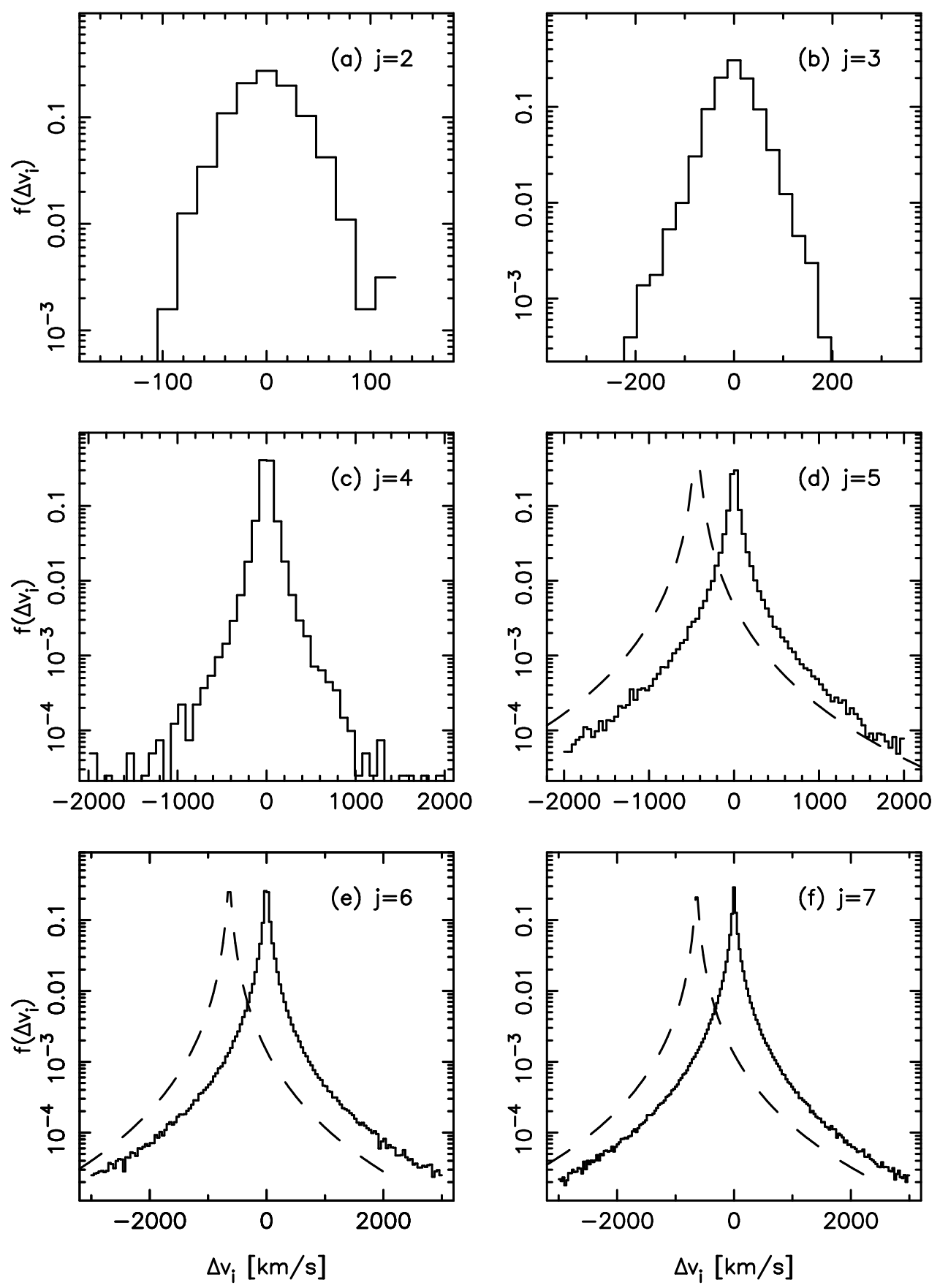

Fig. 11. - The one-point distribution of $\Delta v_{i}$ on scales $j=2-7$ for simulation samples of the SCDM model. The dashed line for $\mathrm{j}=5,6,7$ is the lognormal curve with the same dispersion as the CDM model, which are slightly shifted to left for a clear presentation. 

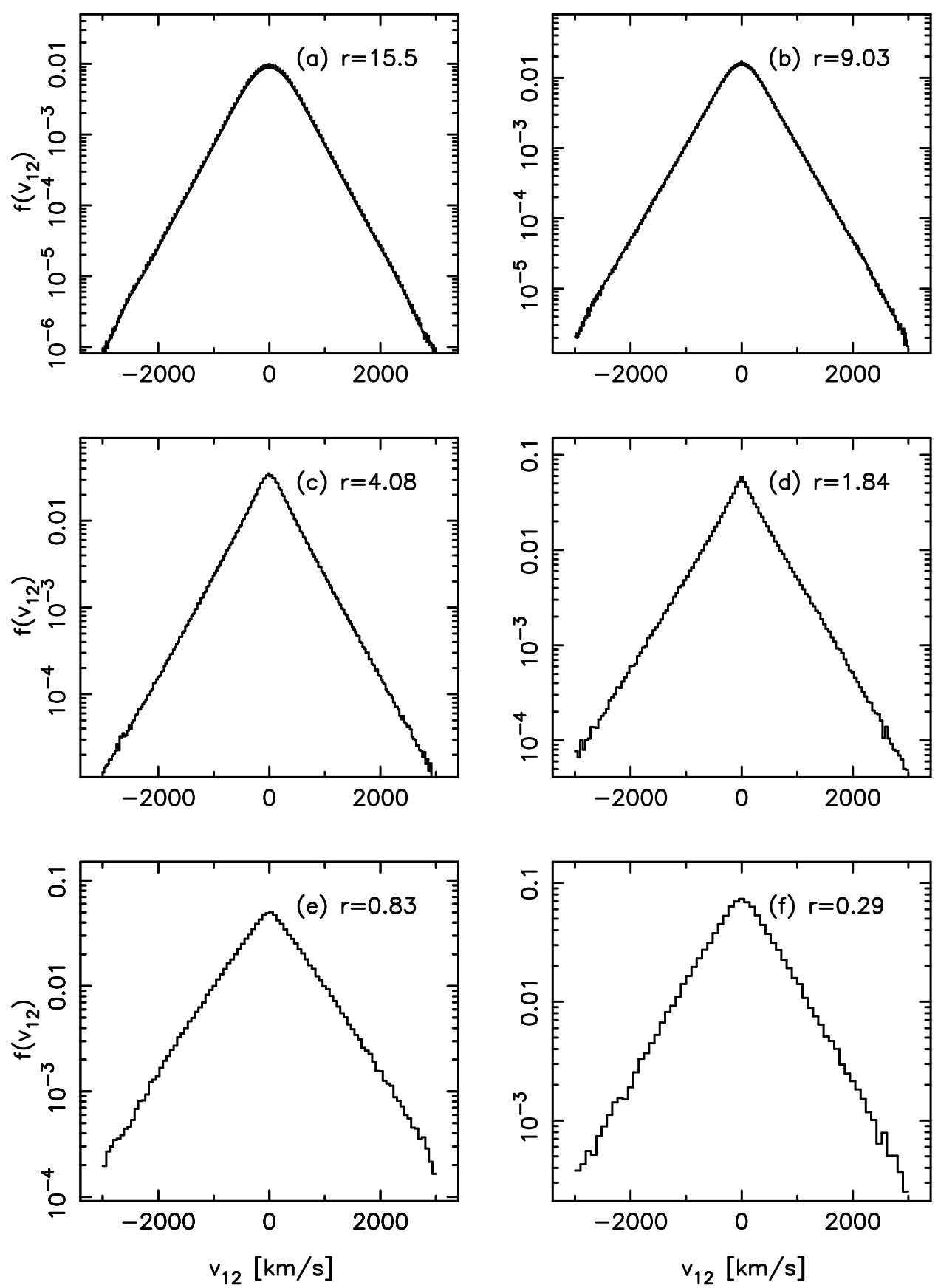

Fig. 12. - The one-point distribution of $v_{12}$ measured by the traditional pair counting method for simulation samples of the SCDM model. $r$ is the distance of the pairs in the unit of $\mathrm{h}^{-1} \mathrm{Mpc}$. 


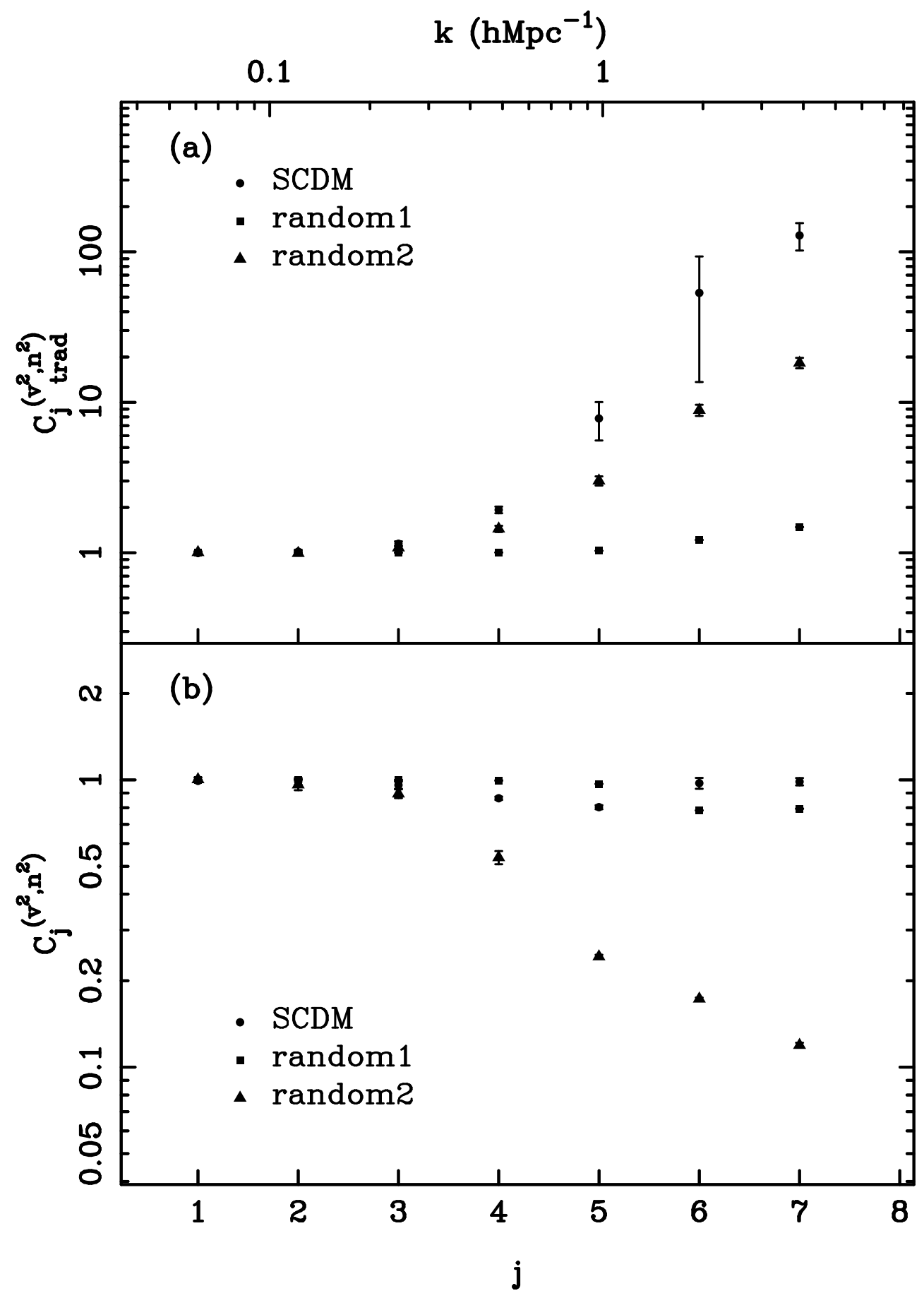

Fig. 13. - The fourth order correlation between the traditional estimated VD and local number density of particles [panel (a)], and the fourth order correlation between the VD and local number density of particles [panel (b)] for model SCDM. The random samples are the same as Fig. 3. The 


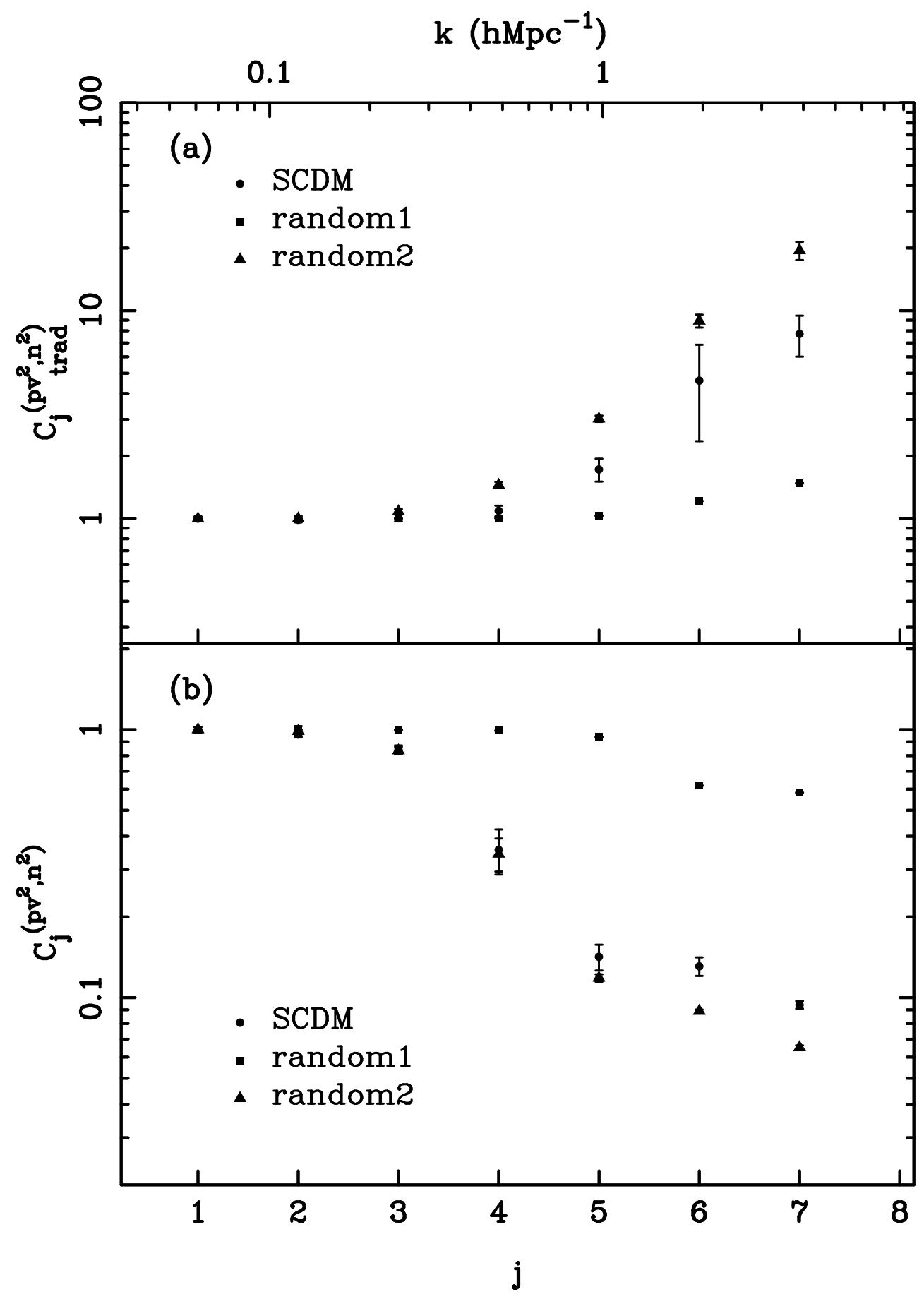

Fig. 14.- The fourth order correlations $C_{j \text { trad }}^{\left(p v^{2}, n^{2}\right)}$ [eq.(57)] (upper panel), and $C_{j}^{\left(p v^{2}, n^{2}\right)}$ [eq.(58)] (lower panel) for model SCDM. The random samples are the same as Fig. 3. The error bars are 1- $\sigma$ variance from 10 realizations. 


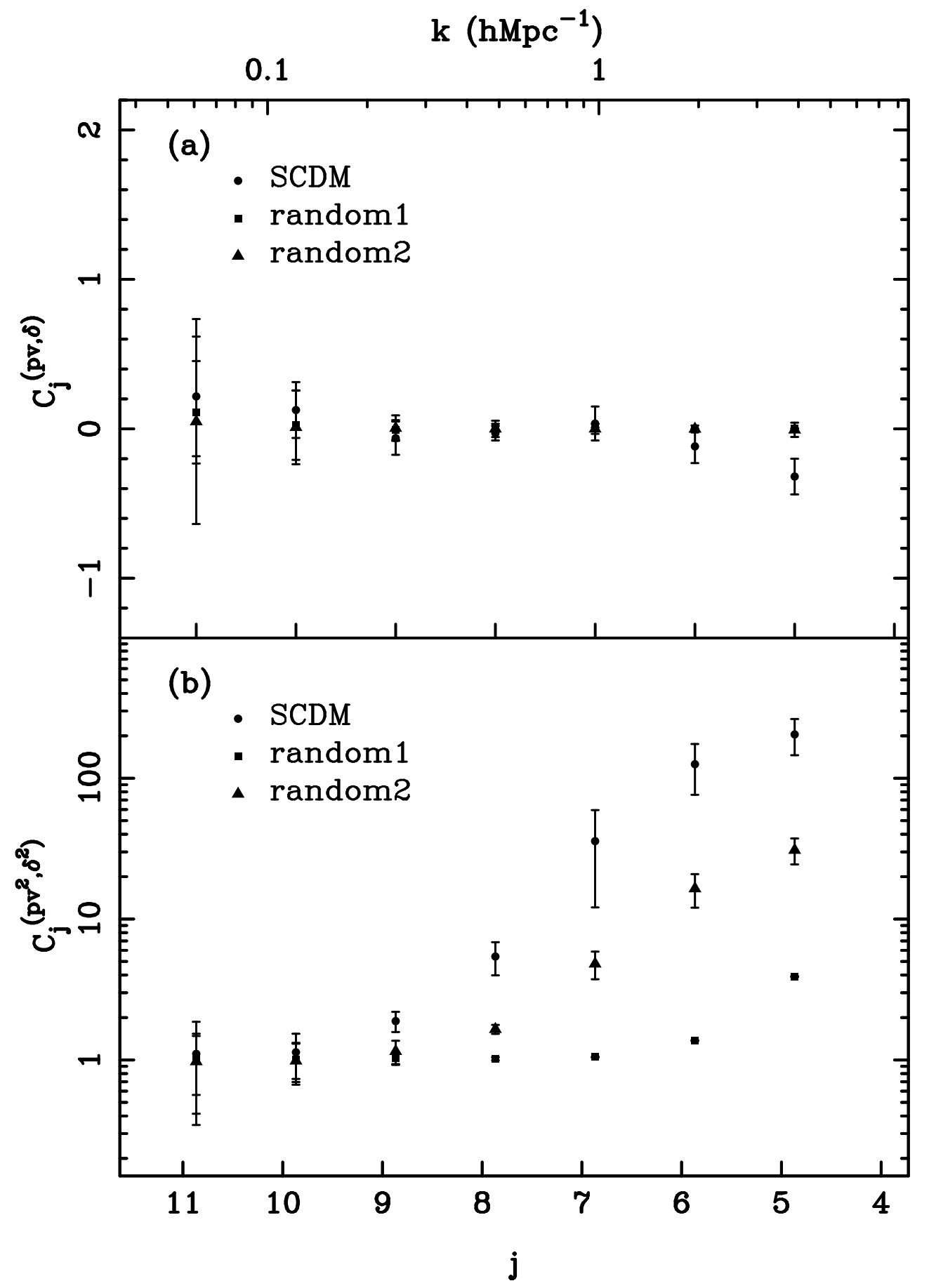

Fig. 15. - The second (upper panel) and fourth (lower panel) order correlations between the PVD and local density fluctuation of particles for model SCDM. The random samples are the same as Fig. 3. The error bars are $1-\sigma$ variance from 10 realizations. 